\title{
Effets combinés des facteurs anthropiques et climatiques sur l'état des populations de trois espèces ligneuses vulnérables
}

\author{
Lassina TRAORE ${ }^{1 *}$, Oumarou SAMBARE ${ }^{2}$, Salfo SAVADOGO ${ }^{3}$, \\ Amadé OUEDRAOGO ${ }^{4}$ et Adjima THIOMBIANO ${ }^{4}$
}

\author{
${ }^{1}$ Unité de Formation et de Recherche en Sciences et Technologies (UFR/ST), Université Norbert ZONGO, BP \\ 376 Koudougou, Burkina Faso. \\ ${ }^{2}$ Institut Des Sciences (IDS), Ouagadougou, Burkina Faso. \\ ${ }^{3}$ Département de Substances Naturelles (DSN), Institut de Recherches en Sciences Appliquées et Technologies \\ (IRSAT), Centre National de la Recherche Scientifique et Technologiques (CNRST), 03 BP 7074 Ouagadougou \\ 03, Burkina Faso. \\ ${ }^{4}$ Laboratoire de Biologie et Écologie Végétales, Unité de Formation et de Recherche en Science de la Vie et de \\ la Terre (UFR/SVT), Université Ouagal Pr Joseph KI-ZERBO, Ouagadougou, 03 BP 7021 Ouagadougou 03, \\ Burkina Faso. \\ *Auteur correspondant ; E-mail : ltraorej@gmail.com
}

\section{RESUME}

Les facteurs climatiques et anthropiques sont à l'origine de la régression de la population de nombreuses espèces ligneuses en zone soudanienne. Anogeissus leiocarpa, Bombax costatum et Detarium microcarpum sont des espèces qui ont une importance socio-économique avérée et donc elles se révèlent être vulnérables. L'évaluation comparée de l'état des peuplements des trois espèces dans les aires protégées et non protégées suivant le gradient climatique permettra de disposer d'informations utiles pour leur gestion durable. La structure des populations a été évaluée à partir d'inventaires forestiers basés sur un échantillonnage aléatoire à travers des parcelles rectangulaires de $1000 \mathrm{~m}^{2}$. Les individus dont le diamètre du tronc à $1,3 \mathrm{~m}$ de hauteur est supérieur ou égal à $5 \mathrm{~cm}$ ont été mesurés. La régénération (individus à D1,3 $\mathrm{m}<5 \mathrm{~cm}$ ) a été comptée par classe de hauteur dans des sous-placettes de $25 \mathrm{~m}^{2}$. Les densités et les surfaces terrières des trois espèces varient significativement suivant le gradient de protection dans chaque secteur climatique. A. leiocarpa et D. microcarpum possèdent de bonnes structures démographiques dans les deux secteurs climatiques soudaniens, indépendamment du type d'utilisation de terres. Seule la densité de B. costatum montre une différence significative le long du gradient climatique entre les aires protégées. L'étude révèle l'importance des aires protégées dans la conservation de ces espèces vulnérables.

(C) 2020 International Formulae Group. All rights reserved.

Mots clés : Burkina Faso, zone soudanienne, aires protégées, aires non protégées, espèces vulnérables, conservation.

\section{ABSTRACT}

Climate and land use types are mainly responsible for the decline of many woody species in Sudanian areas. Anogeissus leiocarpa, Bombax costatum and Detarium microcarpum are high-value but vulnerable species due to the anthropogenic pressure. The compared assessment of the state of the targeted species in the protected 
and unprotected areas along the climatic gradient will provide information that could help implementing sustainable management of these species. The population structures were evaluated from forest inventories based on a random sampling through rectangular plots of $1000 \mathrm{~m}^{2}$. The adult individuals of each species with diameters $\geq 5 \mathrm{~cm}$ at $1.30 \mathrm{~m}$ of heigh were measured. The juvenile individual with diameters that were less than $5 \mathrm{~cm}$ were counted and classified into height classes within plots size of $25 \mathrm{~m}^{2}$. The structural characteristic of the targeted species vary significantly between land uses within each climatic zone. A. leiocarpa and D. microcarpum showed a stable population structure between the two climatic sectors, irrespective of management regimes. Only density of $B$. costatum shows a significant difference in the PAs and along climatic gradient. The study reveals the importance of protected areas for these vulnerable species conservations.

(C) 2020 International Formulae Group. All rights reserved.

Keywords: Burkina Faso, Sudanian zone, protected areas, unprotected areas, vulnerable species, conservation.

\section{INTRODUCTION}

Les ressources naturelles jouent un rôle socio-économique et environnemental considérables dans la vie des populations des pays subsahariens (Bénoit, 2008). En Afrique de l'Ouest, les ligneux contribuent à la satisfaction des besoins en aliments, en bois d'énergie, en bois d'œuvre, en bois de service et en phytothérapie de la majorité des populations (Lykke et al., 2004 ; Ganaba et al., 2004; Ouôba et al., 2006 ; Traoré et al., 2011). La demande sans cesse croissante en produits végétaux est source de pression sur les ressources ligneuses et constitue une cause majeure de menace pour beaucoup d'espèces (Gaoue and Ticktin, 2008 ; Nacoulma et al., 2011). Cette situation est aggravée par la péjoration climatique qui augmente la vulnérabilité des espèces. L'impact de la sécheresse sur les ressources végétales est perceptible depuis les années 1970 (Chappell and Agnew, 2004 ; Dai et al., 2004). Depuis la fin des années 1990, la pluviométrie s'améliore dans les région semi-arides (Dai et al., 2004 ; Nicholson, 2005); cependant, cette amélioration de la pluviométrie annuelle semble être le fait d'une plus forte intensité, qu'un rallongement de la saison des pluies par rapport aux décennies de sécheresse (Ozer et al., 2010). Les manifestations de la péjoration climatique sont la rareté des précipitations, mais également la forte fluctuation de la distribution temporelle et spatiale des précipitations (Diallo et al., 2011) qui constituent des facteurs de contrôle déterminants des écosystèmes soudano- sahéliens et de la modification de la végétation (Ozer et al., 2010).

La surexploitation des terres est également une cause majeure de la dégradation du couvert végétal et de la perte de la biodiversité (Bouko et al., 2007). Cela s'observe en général dans plusieurs zones d'Afrique de l'Ouest (Ariori and Ozer, 2005 ; Sawadogo et al., 2008 ; Diallo et al., 2011) et en particulier au Burkina Faso où la dynamique d'occupation de l'espace par l'agriculture extensive prend aujourd'hui de l'ampleur au détriment des formations végétales plus ou moins naturelles, accentuant du même coup la pression sur les aires protégées. Au Burkina Faso, moins de $10 \%$ de la population a accès à d'autres sources d'énergie que le bois de feu et près de 250000 hectares de forêts sont défrichés annuellement pour satisfaire ces besoins (Devineau, 2010). De nombreuses espèces végétales sont ainsi détruites chaque année malgré leurs importances dans la vie des populations locales. De nombreuses espèces végétales sont ainsi détruites chaque année malgré leurs importances dans la vie des populations locales parmi lesquelles $A$. leiocarpa, D. microcarpum et B. costatum. En effet, la valeur socioéconomique de ces trois espèces est avérée. Les qualités énergétiques du bois d'Anogeissus leiocarpa et de Detarium microcarpum (Bognounou, 2009; Folefack and Abou, 2009 ; Traoré et al., 2011) sont bien connues. Les calices de B. Costatum et les fruits de $D$. microcarpum sont vendus en zone rurales et urbaines du Burkina Faso et génèrent des revenus monétaires (Belem et al., 2008 ; Ouédraogo et al., 2014). Dans plusieurs 
localités au Burkina Faso, B. costatum fait partie des espèces préférées des populations (Mertz et al., 2001) et est menacé à cause d'une surexploitation de ses différents organes (Ouédraogo et al., 2014). La consommation des calices prive les adultes de leur potentiel semencier (Belem et al., 2008). Son intérêt pour les populations locales et ces menaces font de Bombax costatum une espèce à conserver en priorité (Sop et al., 2012). Par ailleurs, les usages médicinal, cosmétique et alimentaire (extraction en huile des graines) de D. microcarpum sont connus et exploités par les populations locales comme moyens de subsistance et sources de revenus (Tiétiambou et al. 2016). A ce titre, ces trois espèces ligneuses sont une source de nombreux produits utilisés sur le plan alimentaire, médicinal et cosmétique par les populations locales. Toutes ces formes d'utilisations constituent alors une pression qui perturbe l'état des populations des espèces utilitaires dans un contexte de prélèvements frauduleux dans les aires protégées et de perturbations climatiques.

De nombreuses études de la végétation de l'Afrique de l'Ouest ont surtout abordé l'influence du climat sur certaines espèces utilitaires (Ouédraogo et al., 2006 ; Weber et al., 2008 ; Ouédraogo and Thiombiano, 2012) et sur certains types de formations végétales (Bognounou et al., 2009; Sambaré et al., 2011). Celles qui ont explicitement abordé la combinaison des facteurs climatiques et anthropiques sur la dynamique de la végétation sont rares (Diallo et al., 2011). Le pire n'est pas l'utilisation abusive des ressources végétales ligneuses mais le manque de données scientifiques susceptibles d'orienter les actions de conservation et de gestion durables de celles qui sont fortement exploitées par les populations locales. C'est dans ce contexte que cette étude se focalise sur les effets combinés des facteurs anthropiques et climatiques sur l'état des populations de trois espèces ligneuses, Anogeissus leiocarpa (DC.) Guill. et Perr., Bombax costatum Pellegr. et Vuillet et Detarium microcarpum Guill. et Perr., dont la vulnérabilité aux pressions anthropiques est révélée par les travaux de Traoré et al. (2011). La présente étude avait pour objectif d'analyser l'état actuel de la population de trois espèces à partir des données d'inventaire de terrain, ce qui permettra d'apporter des solutions pour une meilleure conservation de la population de ces espèces. Pour atteindre cet objectif, nous avons émis l'hypothèse suivante: les types d'utilisation des terres et le gradient climatique influencent significativement la structure des peuplements des trois espèces.

\section{MATERIEL ET METHODES \\ Zone d'étude}

La zone d'étude est la partie Ouest du Burkina Faso, s'étendant entre les secteurs phytogéographiques nord-Soudanien (NS) et sud-Soudanien (SS) (Figure 1). Elle est située entre $9^{\circ} 30^{\prime}-13^{\circ} 30 \mathrm{~N}$ et $5^{\circ} 30^{\prime}-2^{\circ} 30 \mathrm{~W}$. Ces secteurs reçoivent respectivement entre 800$900 \mathrm{~mm}$ et $900-1100 \mathrm{~mm}$ de précipitation moyenne par an (Fontès and Guinko, 1995). Les secteurs phytogéographiques NS et SS sont respectivement situés dans les zones climatiques soudano-sahélienne et soudanienne. Ils sont caractérisés par l'alternance d'une saison sèche, longue de 6 à 7 mois au nord soudanien et de 5 à 6 mois au sud soudanien et d'une saison humide qui dure en moyenne de mai à octobre. Le SS est situé sur le socle précambrien et le NS sur les couvertures sédimentaires (Bognounou, 2009). Selon le système de classification des sols de la FAO (Driessen et al., 2001) les ferrasols sont les plus au SS et les lithosols au NS (Bunasols 2002a, b, c et 2006). Les formations végétales dominantes dans le NS sont les savanes arbustives et les savanes arborées; les savanes arbustives, les savanes arborées et les savanes boisées dans le SS (Traoré et al., 2013). La flore essentiellement soudanienne, est caractérisée au Nord-Soudanien par des espèces ligneuses telles que Vitellaria paradoxa C. F. Gaertn., Lannea microcarpa Engl., Anogeissus leiocarpa Guill. \& Perr., Combretum nigricans Guill. \& Perr.. Au sudSoudanien, les espèces ligneuses remarquables sont Isoberlinia doka Craib \& Stapf, Daniellia oliveri (Rolfe) Hutch. \& Dalziel., Detarium 
microcarpum Guill. \& Perr., Anogeissus leiocarpa Guill. \& Perr. et Pteleopsis suberosa Engl. \& Diels.

La zone d'étude compte 39 zones d'importance écologique réparties en Réserves partielles de faunes, en Forêts classées à vocation faunique et en forêts classées (Belemsobgo et al., 2010) mais celles-ci ne sont pas toujours à l'abri de la pression anthropique (Devineau, 2010). Les zones d'importance écologique qui ont été concernées par cette étude sont présentées dans le paragraphe « échantillonnage » Selon ce dernier auteur, le prélèvement illégal des ligneux et le pâturage en saison sèche, lorsque le fourrage devient rare, sont récurrents dans les aires protégées. Les feux précoces d'aménagement sont également pratiqués dans le but de prévenir l'effet dévastateur des feux tardifs. Toutefois, leur application ne se fait toujours pas à la période indiquée qui est entre octobre et novembre.

La population essentiellement rurale pratique une agriculture dominée par la production extensive des céréales et quelques cultures de rentes comme le coton. En plus de l'agriculture, l'élevage y est également pratiqué. Selon Kagoné (2001), le NS est caractérisé par un agropastoralisme à dominance agricole couplé à une forte densité de populations humaine et animale. Le SS se singularise par une vocation agricole dominée par les cultures pérennes et fait l'objet d'une transhumance en saison sèche.

\section{Les espèces étudiées}

A. leiocarpa (Combretaceae) est une espèce qui s'étend du Sénégal au Soudan et de l'Ethiopie jusqu'en République Démocratique du Congo (Aké assi, 2001 ; Arbonnier, 2002). Elle a une grande plasticité écologique et se rencontre sur presque tous les types de sol, depuis le nord jusqu'au sud du pays (Thiombiano et al., 2006). Selon Arbonnier (2002), A. leiocarpa aurait tendance à préférer les sols argileux. Elle se rencontre dans les savanes, les forêts claires et les galeries forestières soudano-sahélienne à soudano- guinéennes (Arbonnier, 2002). A. leiocarpa est un arbre de hauteur totale : 15 à $30 \mathrm{~m}$ et de son diamètre maximal : $70 \mathrm{~cm}$ selon Arbonnier (2002). L'espèce forme souvent des forêts claires en peuplements quasi monospécifiques. Les feuilles et l'écorce de A. leiocarpa sont employées en médecine traditionnelle dans le traitement de diverses maladies : fièvre, diarrhée, vers intestinaux, fatigue générale (Bognounou, 2009). L'espèce est souvent exploitée pour son bois qui sert à la construction, aux auvres d'art et à la production d'énergie (Lykke et al., 2004 ; Traoré et al., 2011). A. leiocarpa est indicatrice de terres fertiles, propices à l'agriculture (Thiombiano, 2005).

B. costatum (Malvaceae) s'étend du Sénégal au Cameroun jusqu'en République centrafricaine (Arbonnier, 2002). Elle se rencontre à l'état naturel dans les savanes boisées et les forêts claires sahélosoudaniennes à guinéennes (Arbonnier, 2002). Elle est aussi caractéristique des parcs agroforestiers, avec des peuplements étendus de 60-100 individus par hectare (Ouédraogo and Thiombiano, 2012). L'espèce se rencontre sur de nombreux types de sol. Toutefois, elle semble préférer les stations latéritiques ou rocheuses (Arbonnier, 2002). B. costatum est un arbre épineux à fût droit, souvent muni de petits contreforts à la base, de 10-25 m de haut, au port caractéristique dû aux branches quasi droites entre chaque ramification et aux épines coniques disposées sur le tronc et les branches. Cime étalée et ouverte, nettement étagée chez les jeunes sujets (Arbonnier, 2002). La dissémination des graines se fait par le vent et par les animaux (Ouédraogo, 2006). Elle est principalement exploitée pour l'alimentation (fleurs) et pour l'art (bois) (Belem et al., 2008).

D. microcarpum (Fabaceae) s'étend du Sénégal au Soudan jusqu'en Afrique Orientale (Arbonnier, 2002). Au Burkina Faso, son aire de répartition couvre les secteurs sub sahélien, nord soudanien et sud soudanien. Elle se trouve dans les bas-fonds humides et sols frais (Arbonnier, 2002). C'est une espèce des savanes boisées et des forêts claires des zones 
soudano-guinéennes et soudano-sahéliennes (Arbonnier, 2002; Bastide and Ouédraogo, 2008). D. microcarpum est un arbre atteignant 25-30 m de haut, à fût cylindrique, souvent mal conformé, à cime basse et à branches trapues étalées (Arbonnier, 2002). Les fruits de $D$. microcarpum interviennent dans l'alimentation humaine et constituent une importante source de revenus pour les populations (Bastide and Ouédraogo, 2008). Selon la même source, toutes les parties de l'arbre, à l'exclusion du bois, sont utilisées en pharmacopée pour le traitement de maladies diverses, notamment de la méningite, des dysenteries, du paludisme, de la lèpre et autres maladies cutanées. C'est une espèce très exploitée comme bois de feu (Bastide and Ouédraogo, 2008).

\section{Échantillonnage}

Cinq aires protégées (AP) ont été retenues comme sites études : les forêts classées de Sâ (5000 ha) et de Toroba (2700 ha) à Dédougou dans le nord soudanien; la Réserve de Biosphère de la Mare aux Hippopotames (19200 ha) à Bala, la forêt classée de Toumousséni (19608,32 ha) et la forêt classée et réserve de faune de la Comoé-Léraba (125000 ha) dans le sud soudanien (Belemsobgo et al., 2010). Ce choix des AP est dû à la présence effective des individus de la population d'au moins l'une des trois espèces retenues pour cette étude.

Les aires non protégées (ANP) désignent les formations ouvertes aux activités agropastorales, les vieilles jachères; les sites à valeurs agronomiques médiocres (substrats rocheux, gravillonnaires) et ouverts au pâturage et les parcs agroforestiers. Le choix des sites de collectes de données dans les formations ouvertes s'est fait sur la base de la présence de l'une des trois espèces mais aussi en tenant compte des zones où les densités sont moyennes à élevées pour l'espèce. Les deux autres espèces (A. leiocarpa et $D$. microcarpum) sont pour la plupart abattues lors du défrichage des nouveaux champs, ce qui fait qu'elles sont quasi inexistantes dans les parcs agroforestiers. Mais elles sont souvent présentes sur certains sites (substrats rocheux, gravillonnaires) à valeurs agronomiques médiocres et ouverts au pâturage.

\section{Collecte des données au niveau des peuplements adultes}

Dans cette étude, sont considérés comme des populations adultes les individus dont le diamètre à $1,30 \mathrm{~m}$ du sol $(\mathrm{D} 1,30)$ est supérieur ou égal à $5 \mathrm{~cm}$. La structure des populations a été évaluée à partir d'échantillonnages orienté et aléatoires dans des parcelles rectangulaires de $50 \times 20 \mathrm{~m}$, au nombre minimum de 9 par population d'espèce et par type d'utilisation des terres (Tableau 1). Cette taille des parcelles est fréquemment utilisée dans les études sur la végétation de l'Afrique de l'Ouest (Ouédraogo et al., 2006 ; Bognounou et al., 2009). Au sein de chaque parcelle, un inventaire systématique de toutes les tiges adultes de chacune des trois espèces a été réalisé. Le paramètre dendrométrique mesuré est la circonférence à 1,30 m au-dessus du sol. La circonférence du tronc a été mesurée à l'aide d'un mètre ruban souple pour en déduire le diamètre. Cette mesure est de loin préférable à celle obtenue à l'aide d'un compas forestier (Rondeux, 1999) en raison de l'irrégularité de la forme du tronc de ces espèces. Au total, 152 parcelles ont été inventoriées soient 74 dans le nord soudanien et 78 dans le sud soudanien, aussi bien dans les aires protégées que celles non protégées

\section{Mesure de la régénération naturelle}

Les individus de D1,30 inférieur à $5 \mathrm{~cm}$ sont considérés comme des jeunes plants et constituent la régénération naturelle. Celle-ci a été étudiée de façon aléatoire dans des placettes de $5 \mathrm{~m}$ x $5 \mathrm{~m}$ à l'intérieur de grandes placettes de $50 \mathrm{~m}$ x $20 \mathrm{~m}$. Le paramètre dendrométrique mesuré est la hauteur des individus de chaque espèce étudiée. Tous les juvéniles de ces trois espèces ligneuses, quel que soit le mécanisme de régénération, ont été comptés, mesurés et répartis en classe de hauteur. Cinq classes de hauteur ont été retenues : [0-0,5 m], [0,51-1 m], [1,1-1,5 m], [1,51-2 m], $\geq 2 \mathrm{~m}$. 
Analyse des données

\section{Structures démographiques des espèces adultes}

Pour un individu multicaule, avec $t$ tiges ramifiées en dessous de la hauteur de mesure $(\mathrm{D} 1,30 \mathrm{~m})$, le diamètre $d$ est égal à la somme quadratique des diamètres $d s_{i}$ des tiges de l'individu mesuré. La formule du diamètre $d$ s'établit comme suit :

$d=\sqrt{\sum_{i=1}^{t} d s_{i}^{2}}$

La structure horizontale des espèces a été établie en considérant l'ensemble des relevés de chaque population des espèces étudiées et les individus ont été regroupés dans des 10 classes de diamètres d'amplitude $5 \mathrm{~cm}$. Les structures observées ont été ajustées à la distribution théorique de Weibull à trois paramètres (Johnson and Kotz, 1970).

Cette distribution simple et flexible s'ajuste aussi bien parfaitement aux distributions asymétriques positive et négative, qu'à celles normales (Lorimer and krug, 1983 ; Baker et al., 2005). Sa fonction de densité de probabilité, $f$ "suit la formule :

$f(x)=c / b[(x-a) / b]^{c-1} e^{-[x-a / b]}$

où $x$ est le diamètre des arbres ; $a$ est le paramètre de position (seuil minimum de diamètre mesuré); $b$ est le paramètre d'échelle ou de taille; il est lié à la valeur centrale des diamètres des arbres du peuplement considéré; $c$ 'est le paramètre de forme liée à la structure en diamètre considérée.

Pour chaque espèce, les données de diamètre de tous les individus sont utilisées pour l'estimation des paramètres $b$ et $c$ grâce à un algorithme basé sur la méthode du maximum de vraisemblance (Zarnock et al., 1985). La distribution de Weibull peut prendre plusieurs formes selon la valeur du paramètre de forme $c$ (Ryniker et al., 2006). Lorsque $c<$ 1 , la distribution est en "J" renversée ; lorsque $c=1$ la distribution est exponentielle négative. Pour $\mathrm{c}>1$ la distribution est une fonction unimodale. Si $1<\mathrm{c}<3,6$, la distribution est asymétrique positive, lorsque $\mathrm{c}=3,6$, la distribution est approximativement normale et quand $\mathrm{c}>3,6$ la distribution est asymétrique négative.

Une analyse log-linéaire (Caswell, 2001) a été réalisée dans le logiciel SAS version 9.1 pour tester l'adéquation entre la structure observée et la distribution de Weibull. Le modèle global s'écrit :

$\log$ Fréquence $=F+F_{\text {_classe }}+F_{\text {_ajustement }}+\varepsilon$ où $\mathrm{F}=$ Fréquence moyenne des classes $; \mathrm{F} \_$classe $=$ l'écart non aléatoire lié aux différences de fréquences entre les classes; $F_{\text {ajustement }}=1$ 'écart non aléatoire lié aux différences entre les fréquences observées et théoriques; $\varepsilon=1$ 'erreur du modèle. Le résultat du test d'adéquation entre les deux structures (fréquences théorique et observée), est accepté si la valeur de probabilité du test est supérieure à 0,05 .

\section{Dynamique de la population juvénile des trois espèces}

La tendance démographique des trois espèces a été analysée à travers la méthode proposée par Condit et al. (1998) et utilisée par Lykke (1998) et Obiri et al. (2002). Une régression linéaire logarithmique a été calculée pour chaque espèce avec la classe médiane (mi) comme variable indépendante et le nombre d'individus ( $\mathrm{Ni}$ ) de cette classe comme variable dépendante. Pour obtenir les paramètres de stabilité, $\mathrm{N} i$ a été soumis à une transformation logarithmique en utilisant $\ln (\mathrm{N} i+1)$ à cause du faite que certaines classes de diamètre ne contiennent pas d'individus (Obiri et al., 2002; Sop et al., 2010). La régression a été exécutée entre $\ln (\mathrm{N} i+1)$ et $\ln (\mathrm{mi})$. Les valeurs des pentes (a) issues des équations de régression et de corrélation $r^{2}$ sont considérées comme des indicateurs de la stabilité de la population de chaque espèce (Tabuti, 2007; Obiri et al., 2002). L'interprétation de la forme de la structure de la population de chaque espèce est celle utilisée par Shackleton (1993), Everard et al. (1995) et Obiri et al. (2002). Une pente négative indique que les individus de petite taille sont plus nombreux. Une pente nulle indique que les effectifs de petite et de grande taille sont égaux. La valeur positive de la pente indique que les individus de grande taille sont les plus nombreux. 
Dans le but de déterminer la stabilité de la structure de la population de chaque espèce, le quotient entre les effectifs successifs des classes de diamètre a été calculé. Les résultats obtenus sont habituellement présentés sous la forme de graphique (Venter and Witkowski, 2010). Lorsque les valeurs des quotients sont constantes entre les classes de diamètre successives, la population est dite stable, tandis que celles qui sont variables indiquent une population instable (Botha et al., 2004 ; Mwavu and Witkowski, 2009 ; Sop et al., 2010).

Par ailleurs, l'indice de Permutation (PI) proposé par Wiegand et al. (2000) et Botha et al. (2004) a été utilisée pour déterminer la stabilité du peuplement de chaque espèce. PI mesure le degré de déviation par rapport à une structure stable attendue au sein d'une population non perturbée. Le principe est basé sur le fait que pour une population stable non perturbée, les effectifs des classes de diamètre décroissent régulièrement, de la première à la dernière classe de diamètre. L'expression de PI selon Botha et al. (2004) est :

$\mathrm{PI}=\sum_{i=1}^{\mathrm{k}}|\mathrm{J} i-\mathrm{i}| ; \mathrm{J} i=1,2, \ldots \ldots, \mathrm{k}$ où $\mathrm{J} i$ est le rang de classe de diamètre $i$ ( $i=1$ pour la plus petite classe), avec le plus grand rang $(\mathrm{Ji}=1)$ attribué à la classe de diamètre la plus fréquente. $\mathrm{PI}=0$ indique une population stable non perturbée et lorsque PI > 0 , la population est perturbée (Venter and Witkowski, 2010). Pour une meilleure comparaison de la structure des juvéniles de chaque espèce, les valeurs de quotient de chaque espèce dans les AP et les ANP ont été combinées pour réaliser un seul graphique pour une même espèce à l'intérieur d'un même secteur phytogéographique.

\section{Tests statistiques}

Le test $t$ a été utilisé pour comparer les moyennes des paramètres structurels des populations adultes et juvéniles des espèces entre les types d'utilisation des terres et entre les deux secteurs phytogéographiques. Du fait de la variabilité de forme des structures des espèces, le test de Kolmogorov-Smirnov a été utilisé à une significativité de $5 \%$ pour comparer la tendance démographique (les pentes) en termes de rajeunissement des peuplements entre les types d'utilisations des terres et entre les deux secteurs phytogéographiques.

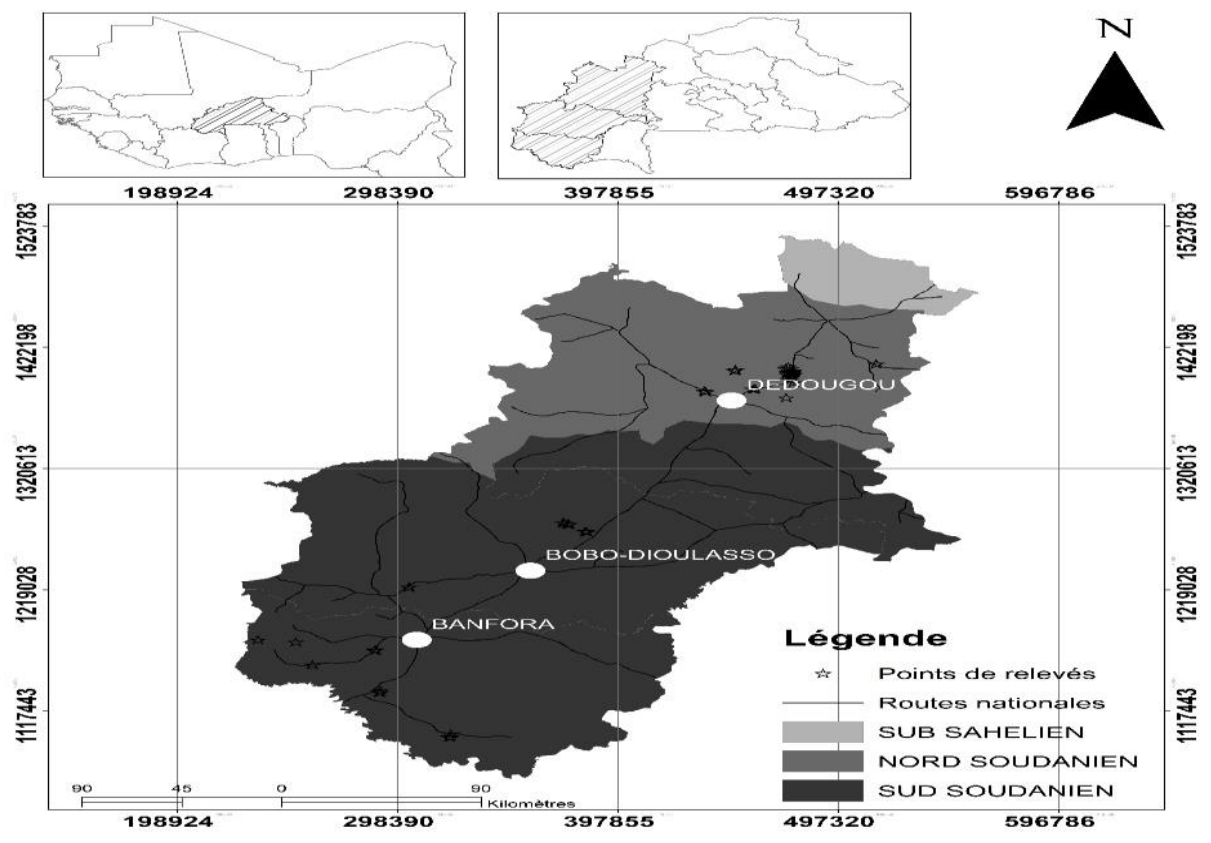

Figure 1 : Localisation de la zone d'étude. 


\section{RESULTATS}

\section{Caractéristiques démographique et dendrométrique des arbres}

Les paramètres démographiques (densités moyennes) des trois espèces varient significativement suivant les types d'utilisation des terres (Tableau 1). Les plus fortes valeurs de densités moyennes des trois espèces sont enregistrées dans les AP. Dans les AP du NS, les densités moyennes de $A$. leiocarpa, de $B$. costatum et de $D$. microcarpum sont respectivement de 338,18 $\pm 65,54 \mathrm{ind} / \mathrm{ha}$; de $94,73 \pm 30,94 \mathrm{ind} / \mathrm{ha}$ et de $374 \pm 183,67 \mathrm{ind} / \mathrm{ha}$. Dans les ANP du NS, les densités moyennes de $A$. leiocarpa, de $B$. costatum et de D. microcarpum sont respectivement de $268 \pm$ $71,3 \mathrm{ind} / \mathrm{ha}$, de $68,67 \pm 15,05 \mathrm{ind} / \mathrm{ha}$ et de $176,67 \pm 100,49$ ind/ha. Dans les AP du SS, les densités moyennes respectives des trois espèces sont : 407,5 $\pm 214,39 \mathrm{ind} / \mathrm{ha}, 255 \pm$ $158,15 \mathrm{ind} / \mathrm{ha}$ et $300 \pm 86,89 \mathrm{ind} / \mathrm{ha}$. Dans les ANP du SS, les densités moyennes de $A$. leiocarpa, de $B$. costatum et de $D$. microcarpum sont respectivement : 204,17 \pm $81,06 \mathrm{ind} / \mathrm{ha}, 76,42 \pm 22,39 \mathrm{ind} / \mathrm{ha}$ et $210 \pm$ $82,25 \mathrm{ind} / \mathrm{ha}$.

Les caractéristiques dendrométriques varient significativement suivant les types d'utilisation des terres $(p<0,05)$ (Tableau 1). Les valeurs des surfaces terrières les plus élevées sont observées dans les AP, des deux secteurs phytogéographiques (NS et SS), excepté la surface terrière de $B$. costatum qui est significativement plus élevée dans les ANP que dans les AP du NS. Dans les AP du NS, les surfaces terrières de A. leiocarpa, de $B$. costatum et de $D$. microcarpum sont respectivement de $10,74 \pm 3,63 \mathrm{~m}^{2} / \mathrm{ha}$, de 10,11 $\pm 13,55 \mathrm{~m}^{2} /$ ha et de $6,97 \pm 3,28 \mathrm{~m}^{2} / \mathrm{ha}$. Dans les ANP du NS, les valeurs respectives des surfaces terrières (de A. leiocarpa, de $B$. costatum et de D. microcarpum) sont : 6,83 \pm $2,43 \mathrm{~m}^{2} / \mathrm{ha}, 24,1 \pm 18,5 \mathrm{~m}^{2} /$ ha et $2,64 \pm 2,08$ $\mathrm{m}^{2} / \mathrm{ha}$. En ce qui concerne les AP du SS, les surfaces terrières de A. leiocarpa, B. costatum et $D$. microcarpum sont respectivement $8,97 \pm 2,41 \mathrm{~m}^{2} / \mathrm{ha}, 9,85 \pm 4,54 \mathrm{~m}^{2} /$ ha et $3,87 \pm$ $2,13 \mathrm{~m}^{2} / \mathrm{ha}$. Les valeurs respectives des surfaces terrières des trois espèces dans les
ANP du même secteur phytogéographique sont $5,57 \pm 4,39 \mathrm{~m}^{2} / \mathrm{ha}, 5,32 \pm 6,39 \mathrm{~m}^{2} /$ ha et $2,4 \pm$ $0,95 \mathrm{~m}^{2} / \mathrm{ha}$.

En considérant le gradient climatique, dans les ANP des secteurs phytogéographiques NS et SS les densités moyennes de A. leiocarpa (NS : $268 \pm 71,3 \mathrm{ind} / \mathrm{ha}$; SS : 204,16 $\pm 81,06$ ind/ha ; $\mathrm{p}=0,066$ ), de B. costatum (NS : 68,67 $\pm 15,05 \mathrm{ind} / \mathrm{ha} ; \mathrm{SS}: 76,42 \pm 22,39 \mathrm{ind} / \mathrm{ha} ; \mathrm{p}=$ 0,280 ) et de $D$. microcarpum (NS : 176,67 \pm $100,49 \mathrm{ind} / \mathrm{ha} ; \mathrm{SS}: 210 \pm 82,25 \mathrm{ind} / \mathrm{ha} ; \mathrm{p}=$ $0,403)$ ne présentent aucune différence significative. Dans les AP du NS et du SS, la densité moyenne de $B$. costatum (NS : 94,73 \pm 30,97 ind/ha; SS : $255 \pm 158,15 \mathrm{ind} / \mathrm{ha} ; \mathrm{p}=$ $0,000)$ présente une différence significative suivant le gradient climatique. La plus forte densité est observée au SS (Tableau 2).

Pour ce qui est de la surface terrière des trois espèces dans les ANP, seule $B$. costatum (NS : $24,1 \pm 18,5 \mathrm{~m}^{2} / \mathrm{ha} ; \mathrm{SS}: 5,32 \pm 6,39$ $\left.\mathrm{m}^{2} / \mathrm{ha} ; \mathrm{p}=0,001\right)$ présente une différence significative. La plus forte valeur de la surface terrière est observée au NS. Les deux autres espèces, A. leiocarpa (NS : $6,83 \pm 2,43 \mathrm{~m}^{2} / \mathrm{ha}$; SS : $\left.5,57 \pm 4,39 \mathrm{~m}^{2} / \mathrm{ha} ; \mathrm{p}=0,424\right)$ et $D$. microcarpum (NS : $2,64 \pm 2,08 \mathrm{~m}^{2} / \mathrm{ha} ; \mathrm{SS}: 2,4$ $\pm 0,95 \mathrm{~m}^{2} / \mathrm{ha} ; \mathrm{p}=0,72$ ) ne montrent aucune différence significative dans les ANP des secteurs phytogéographiques NS et SS (Tableau 2). Dans les AP, les surfaces terrières de A. leiocarpa (NS : 10,74 $\pm 3,63 \mathrm{~m} 2 / \mathrm{ha}$; SS : $\left.8,97 \pm 2,41 \mathrm{~m}^{2} / \mathrm{ha} ; \mathrm{p}=0,178\right)$ et de $B$. costatum (NS : $10,11 \pm 13,55 \mathrm{~m}^{2} /$ ha $; \mathrm{SS}: 9,85 \pm 4,54$ $\left.\mathrm{m}^{2} / \mathrm{ha} ; \mathrm{p}=0,946\right)$ ne montrent aucune différence significative du nord au sud. Par contre, la surface terrière de $D$. microcarpum (NS : $6,97 \pm 3,28 \mathrm{~m}^{2} / \mathrm{ha} ; \mathrm{SS}: 3,87 \pm 2,13 \mathrm{~m}^{2} / \mathrm{ha}$; $\mathrm{p}=0,012)$ diffère significativement du nord au sud (Tableau 2).

\section{Structure en classes de diamètre et tendances démographiques des espèces}

Les structures en classe de diamètre d'A. leiocarpa et de D. microcarpum révèlent des populations stables selon les valeurs du paramètre de forme $(\mathrm{c}<1)$ de la distribution de Weibull dans les AP et les ANP des deux secteurs climatiques (Figure 2). Les résultats 
du test lié à la différence entre les deux distributions des deux espèces sans distinction de types d'utilisation des terres ne sont pas significatifs $(p>0,05)$ (Tableau 6), ce qui indique une concordance entre les structures démographiques observées et celles déduites de la distribution théorique de Weibull. Les valeurs des indicateurs de stabilité d'A. leiocarpa (AP : pente $=-1,24 \pm 0,36 ; \mathrm{r}^{2}=$ 0,$67 ;$ ANP : pente $\left.=-1,20 \pm 0,46 ; r^{2}=0,66\right)$ et de D. microcarpum (AP : pente $=-1,27 \pm 0,51$; $\mathrm{r}^{2}=0,68 ;$ ANP : pente $=-1,08 \pm 0,30 ; \mathrm{r}^{2}=$ $0,73)$ dans le NS d'une part, et celles des mêmes espèces (respectivement) (AP : pente $=$ $-1,56 \pm 0,42 ; r^{2}=0,79 ;$ ANP $:$ pente $=-1,08 \pm$ 0,$\left.68 ; r^{2}=0,65\right)$ et $(\mathrm{AP}:$ pente $=-1,64 \pm 0,28$; $r^{2}=0,80 ;$ ANP : pente $=-1,25 \pm 0,29 ; r^{2}=$ 0,73 ) dans le SS d'autre part montrent que leur tendance démographique obéit à un rajeunissement de leurs populations (Tableau $3)$. Le test de comparaison de pente révèle une différence significative seulement pour $D$. microcarpum dans le SS indiquant une proportion plus importante de jeunes individus dans les AP que les ANP (Tableau 3).

Les fluctuations moyennes de quotient affichées dans le NS, aussi bien dans les AP que les ANP, pour A. leiocarpa et $D$. microcarpum révèlent une relative stabilité des effectifs de juvéniles de A. leiocarpa. Les effectifs de juvéniles de D. microcarpum seraient stables uniquement dans les aires protégées (Figure 3). Dans le SS, les variations du quotient des effectifs successifs des classes de diamètre sont faibles; ce qui présage une stabilité des effectifs de juvéniles au niveau des différentes classes de diamètre dans ce secteur SS. La valeur de l'indice de permutation (PI = 0 ) obtenue dans les deux secteurs (NS et SS) pour A. leiocarpa et $D$. microcarpum dans les deux types d'utilisations des terres traduit la stabilité de leurs populations (Tableau 3 ).

Pour B. costatum, les valeurs $\mathrm{du}$ paramètre de forme $\mathrm{c}>1$ de la distribution de Weibull révèlent une dynamique perturbée dans les AP et les ANP des deux secteurs climatiques (Figure 2). Les valeurs du $\mathrm{r}^{2}$ et de la pente (Tableau 3) montrent que les populations adultes de cette espèce sont perturbées. Ce qui est confirmé par l'indice de permutation $(\mathrm{P}>0)$. Cependant, dans le NS, il y a une différence significative au niveau de la pente, traduisant une proportion plus importante de jeunes individus dans les AP que dans les ANP.

En considérant le gradient climatique, les tendances démographiques (pentes) ne varient pas d'un secteur phytogéographique à un autre au niveau des AP. Mais dans l'ensemble, on note une tendance au rajeunissement des populations des trois espèces des AP. Cela se traduit par des valeurs absolues de pentes légèrement plus élevées au sud qu'au nord dans les AP (Tableau 4). On note une différence significative au niveau de la pente de $B$. costatum du nord au sud soudanien dans les ANP (Tableau 4). Cela traduit la jeunesse des populations de $B$. costatum dans le sud soudanien par rapport au nord soudanien.

\section{Caractéristiques démographiques de la régénération naturelle}

Dans le NS, les densités moyennes de $A$. leiocarpa (AP :12981,82 $\pm 9612,26 \mathrm{ind} / \mathrm{ha}$; ANP : 4945,45 $\pm 3723,53 \mathrm{ind} / \mathrm{ha} ; \mathrm{p}=0,017$ ), de B. costatum (AP : $2120 \pm 2164,20 \mathrm{ind} / \mathrm{ha}$; ANP : 742,85 $\pm 644,12$ ind/ha $; \mathrm{p}=0 ; 028)$ et de D. microcarpum (AP : $20400 \pm 12168,05$ ind/ha ; ANP : $5000 \pm 3806,76$ ind/ha ; $\mathrm{p}=$ 0,002 ) varient significativement des AP aux ANP (Tableau 6).

Dans le SS, les densités moyennes de $A$. leiocarpa (AP : 11983,33 \pm 9490,30 ind/ha ; ANP : $9094,73 \pm 7092,83 ; \mathrm{p}=0,276)$ de $B$. costatum (AP : $5700 \pm 3411,35$ ind/ha ; ANP : $2900 \pm 3714,06 \mathrm{ind} / \mathrm{ha} ; \mathrm{p}=0,078)$ ne montrent aucune différence significative entre les AP et les ANP (Tableau 6). La densité moyenne de D. microcarpum (AP : $29857,14 \pm 18745,15$ ind/ha; ANP : 9257,14 $\pm 6942,14 ; p=0,034)$ ne varie pas significativement des AP aux ANP (Tableau 6).

A. leiocarpa et D. microcarpum possèdent de bonnes structures démographiques caractérisées par la présence d'individus dans toutes les classes de hauteurs (Figure 4). Les valeurs des paramètres de 
stabilité (pente et $\mathrm{r}^{2}$ ) montrent que les populations juvéniles d'A. leiocarpa et de $D$. microcarpum sont stables. Cette stabilité est confirmée par la valeur de l'indice de permutation $(P=0)$ des deux espèces dans les deux secteurs phytogéographiques (Tableau 6). La population juvénile de $B$. costatum a des caractéristiques similaires dans les AP du SS.

En comparant séparément les AP et les ANP suivant le gradient climatique, on a les résultats suivants :

Dans les AP, les densités moyennes de A. leiocarpa (NS : $12981,82 \pm 9612,26 \mathrm{ind} / \mathrm{ha}$; SS : 11983,33 $\pm 9490,3$ ind $/ \mathrm{ha} ; \mathrm{p}=0,775)$ et de D. microcarpum (NS : $20400 \pm 12168,05$ ind/ha ; SS : 20857,14 $\pm 18745,15$ ind/ha ; p =
$0,936)$ ne varient pas significativement entre le NS et SS (Tableau 7). La densité moyenne de B. costatum (NS : $2120 \pm 2164,2 \mathrm{ind} / \mathrm{ha}$; SS : $5700 \pm 3411,35 ; \mathrm{p}=0,000)$ varie du NS au SS. La densité moyenne la plus élevée se trouve au SS.

Dans les ANP, on observe la même tendance. Les densités moyennes de $A$. leiocarpa (NS : 4945,45 \pm 3723,53 ind/ha; SS :9094,74 $\pm 7092,83 ; \mathrm{p}=0,083)$ et de $D$. microcarpum (NS : $5000 \pm 3806,76 \mathrm{ind} / \mathrm{ha}$; SS : $9257,14 \pm 6942,14 \mathrm{ind} / \mathrm{ha} ; \mathrm{p}=0,127)$. Seule la densité moyenne de $B$. costatum (NS : $742,85 \pm 644,16$ ind/ha; SS : $2900 \pm 3714,06$ ind/ha; $\mathrm{p}=0,043$ ) varie significativement $\mathrm{du}$ NS au SS.
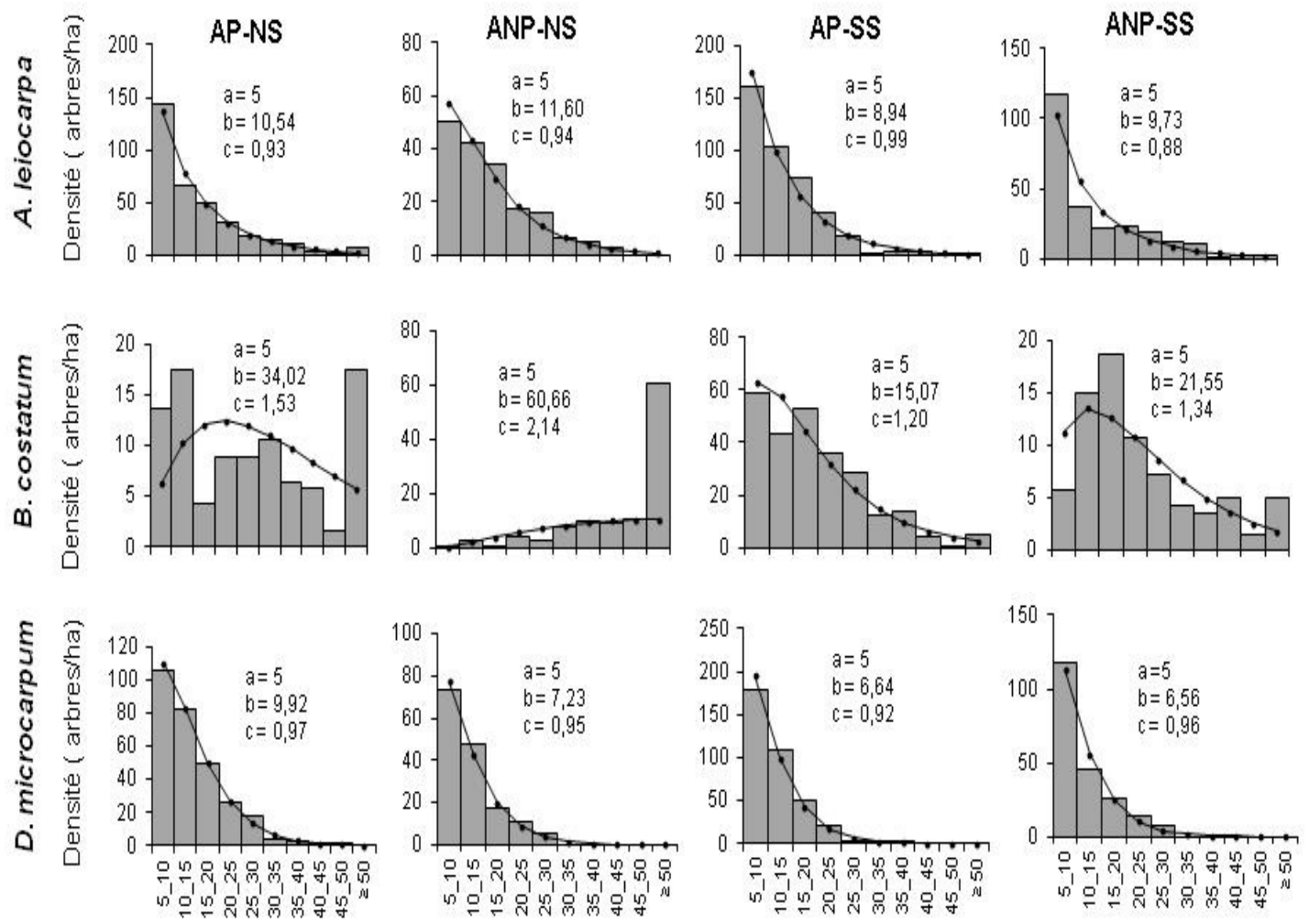

Classes de diamètre $(\mathrm{cm})$

$\square$ Densité observée $\rightarrow$ Weibull

Figure 2 : Structures démographiques de trois espèces (A. leiocarpa, B. costatum et $D$. microcarpum) dans les aires protégées (AP) et dans les aires non protégées (ANP) du Nord-Soudanien (NS) et du Sud-Soudanien (SS). 


\section{TRAORE et al. / Int. J. Biol. Chem. Sci. 14(5): 1763-1785, 2020}

Tableau 1 : Comparaison de la densité et de la surface terrière de trois espèces entre deux types d'utilisation des terres (AP et ANP) en fonction des secteurs phytogéographiques.

\begin{tabular}{|c|c|c|c|c|c|c|c|c|c|c|c|}
\hline secteurs & Espèces & statut & $\begin{array}{l}\text { Nb. } \\
\text { relevé }\end{array}$ & Densité moyenne (ind/ha) & ET & valeur $\mathbf{t}$ & $\mathbf{p}$ & $\mathbf{G}$ & ET & valeur $\mathbf{t}$ & $\mathbf{p}$ \\
\hline \multirow[t]{6}{*}{ NS } & Anogeissus leiocarpa & $\mathrm{AP}$ & 11 & 338,18 & 65,54 & $-2,35$ & $0,029 *$ & 10,74 & 3,63 & $-2,86$ & $0,009 *$ \\
\hline & & ANP & 10 & 268 & 71,3 & & & 6,83 & 2,43 & & \\
\hline & Bombax costatum & $\mathrm{AP}$ & 19 & 94,73 & 30,97 & $-2,98$ & $0,005^{*}$ & 10,11 & 13,55 & 2,54 & $0,015^{*}$ \\
\hline & & ANP & 15 & 68,67 & 15,05 & & & 24,1 & 18,5 & & \\
\hline & Detarium microcarpum & $\mathrm{AP}$ & 10 & 374 & 183,67 & $-2,85$ & $0,010^{*}$ & 6,97 & 3,28 & $-3,38$ & $0,003^{*}$ \\
\hline & & ANP & 9 & 176,67 & 100,49 & & & 2,64 & 2,08 & & \\
\hline \multirow[t]{6}{*}{ SS } & Anogeissus leiocarpa & $\mathrm{AP}$ & 12 & 407,5 & 214,39 & $-3,07$ & $0,005^{*}$ & 8,97 & 2,41 & $-2,35$ & $0,028^{*}$ \\
\hline & & ANP & 12 & 204,17 & 81,06 & & & 5,57 & 4,39 & & \\
\hline & Bombax costatum & $\mathrm{AP}$ & 14 & 255 & 158,15 & $-4,183$ & $0,000^{*}$ & 9,85 & 4,54 & $-2,16$ & $0,040^{*}$ \\
\hline & & ANP & 14 & 76,42 & 22,39 & & & 5,32 & 6,39 & & \\
\hline & Detarium microcarpum & $\mathrm{AP}$ & 13 & 300 & 86,89 & $-2,712$ & $0,012 *$ & 3,87 & 2,13 & $-2,26$ & $0,032 *$ \\
\hline & & ANP & 13 & 210 & 82,25 & & & 2,4 & 0,95 & & \\
\hline
\end{tabular}

$\mathrm{NS}=$ nord soudanien $; \mathrm{SS}=$ sud soudanien $; \mathrm{AP}=$ aire protégée $; \mathrm{ANP}=$ aire non protégée $; \mathrm{Nb}=$ nombre $; \mathrm{ET}=$ écart type $; \mathrm{G}=$ surface terrière $;$ ind/ha $=$ individus/ha

*valeur significative 
L. TRAORE et al. / Int. J. Biol. Chem. Sci. 14(5): 1763-1785, 2020

Tableau 2 : Comparaison de la densité et de surface terrière des trois espèces sur chaque type d'utilisation des terres suivant le gradient climatique.

\begin{tabular}{|c|c|c|c|c|c|c|c|c|c|c|}
\hline Statut & Espèces & Secteurs & Densité moyenne (ind/ha) & ET & valeur $\mathbf{t}$ & $\mathbf{p}$ & $\mathbf{G}$ & ET & valeur $t$ & $\mathbf{p}$ \\
\hline \multirow[t]{6}{*}{ ANP } & \multirow{2}{*}{ Anogeissus leiocarpa } & NS & 268 & 71,3 & \multirow[b]{2}{*}{1,94} & \multirow[b]{2}{*}{0,066} & 6,83 & 2,43 & \multirow[b]{2}{*}{0,81} & \multirow[b]{2}{*}{0,424} \\
\hline & & SS & 204,16 & 81,06 & & & & & & \\
\hline & \multirow[t]{2}{*}{ Bombax costatum } & NS & 68,67 & 15,05 & \multirow{2}{*}{$-1,10$} & \multirow{2}{*}{0,280} & 24,1 & 18,5 & \multirow{2}{*}{3,59} & \multirow{2}{*}{$0,001 *$} \\
\hline & & SS & 76,42 & 22,39 & & & 5,32 & 6,39 & & \\
\hline & \multirow[t]{2}{*}{ Detarium microcarpum } & NS & 176,67 & 100,49 & \multirow{2}{*}{$-0,85$} & \multirow{2}{*}{0,403} & 2,64 & 2,08 & \multirow{2}{*}{0,36} & \multirow{2}{*}{0,72} \\
\hline & & SS & 210 & 82,25 & & & 2,4 & 0,95 & & \\
\hline \multirow[t]{6}{*}{ AP } & \multirow[t]{2}{*}{ Anogeissus leiocarpa } & NS & 338,18 & 65,54 & \multirow[t]{2}{*}{$-1,02$} & \multirow[t]{2}{*}{0,315} & 10,74 & 3,63 & \multirow[t]{2}{*}{1,39} & \multirow[t]{2}{*}{0,178} \\
\hline & & SS & 407,50 & 214,39 & & & 8,97 & 2,41 & & \\
\hline & \multirow[t]{2}{*}{ Bombax costatum } & NS & 94,73 & 30,97 & \multirow[t]{2}{*}{$-4,32$} & \multirow[t]{2}{*}{$0,000^{*}$} & 10,11 & 13,55 & \multirow[t]{2}{*}{0,06} & \multirow[t]{2}{*}{0,946} \\
\hline & & SS & 255 & 158,15 & & & 9,85 & 4,54 & & \\
\hline & \multirow[t]{2}{*}{ Detarium microcarpum } & NS & 374 & 183,67 & \multirow[t]{2}{*}{1,28} & \multirow[t]{2}{*}{0,213} & 6,97 & 3,28 & \multirow[t]{2}{*}{2,74} & \multirow[t]{2}{*}{$0,012 *$} \\
\hline & & SS & 300 & 86,89 & & & 3,87 & 2,13 & & \\
\hline
\end{tabular}


Tableau 3 : Comparaison des indicateurs de stabilité (pente et $\mathrm{r}^{2}$ ) de trois espèces entre les deux types d'utilisation des terres dans chaque secteur phytogéographique.

\begin{tabular}{|c|c|c|c|c|c|c|c|}
\hline secteurs & Espèces & statut & pentes & ET & $\mathbf{p}$ & $\mathbf{r}^{2}$ & PI \\
\hline \multirow[t]{6}{*}{ NS } & \multirow[t]{2}{*}{ Anogeissus leiocarpa } & $\mathrm{AP}$ & $-1,24$ & 0,36 & \multirow{2}{*}{$\mathrm{p}>0,10$} & 0,67 & 0 \\
\hline & & ANP & $-1,20$ & 0,46 & & 0,66 & 0 \\
\hline & \multirow[t]{2}{*}{ Bombax costatum } & $\mathrm{AP}$ & $-0,19$ & 0,52 & \multirow{2}{*}{$\mathrm{p}<0,005^{*}$} & 0,29 & 22 \\
\hline & & ANP & 0,39 & 0,29 & & 0,28 & 50 \\
\hline & \multirow[t]{2}{*}{ Detarium microcarpum } & $\mathrm{AP}$ & $-1,27$ & 0,51 & \multirow{2}{*}{$\mathrm{p}<0,10$} & 0,68 & 0 \\
\hline & & ANP & $-1,08$ & 0,30 & & 0,73 & 0 \\
\hline \multirow[t]{6}{*}{ SS } & \multirow[t]{2}{*}{ Anogeissus leiocarpa } & $\mathrm{AP}$ & $-1,56$ & 0,42 & \multirow{2}{*}{$\mathrm{p}<0,10$} & 0,79 & 0 \\
\hline & & ANP & $-1,08$ & 0,60 & & 0,65 & 0 \\
\hline & \multirow[t]{2}{*}{ Bombax costatum } & $\mathrm{AP}$ & $-0,79$ & 0,51 & \multirow{2}{*}{$\mathrm{p}<0,10$} & 0,46 & 8 \\
\hline & & ANP & $-0,23$ & 0,37 & & 0,23 & 19 \\
\hline & \multirow[t]{2}{*}{ Detarium microcarpum } & $\mathrm{AP}$ & $-1,64$ & 0,28 & \multirow{2}{*}{$\mathrm{p}<0,025^{*}$} & 0,80 & 0 \\
\hline & & ANP & $-1,25$ & 0,29 & & 0,73 & 0 \\
\hline
\end{tabular}

Les valeurs de ET, $\mathrm{p}, \mathrm{r}^{2}$ et PI représentent respectivement les écarts types, le seuil de probabilité, le coefficient de corrélation et l'indice de permutation.
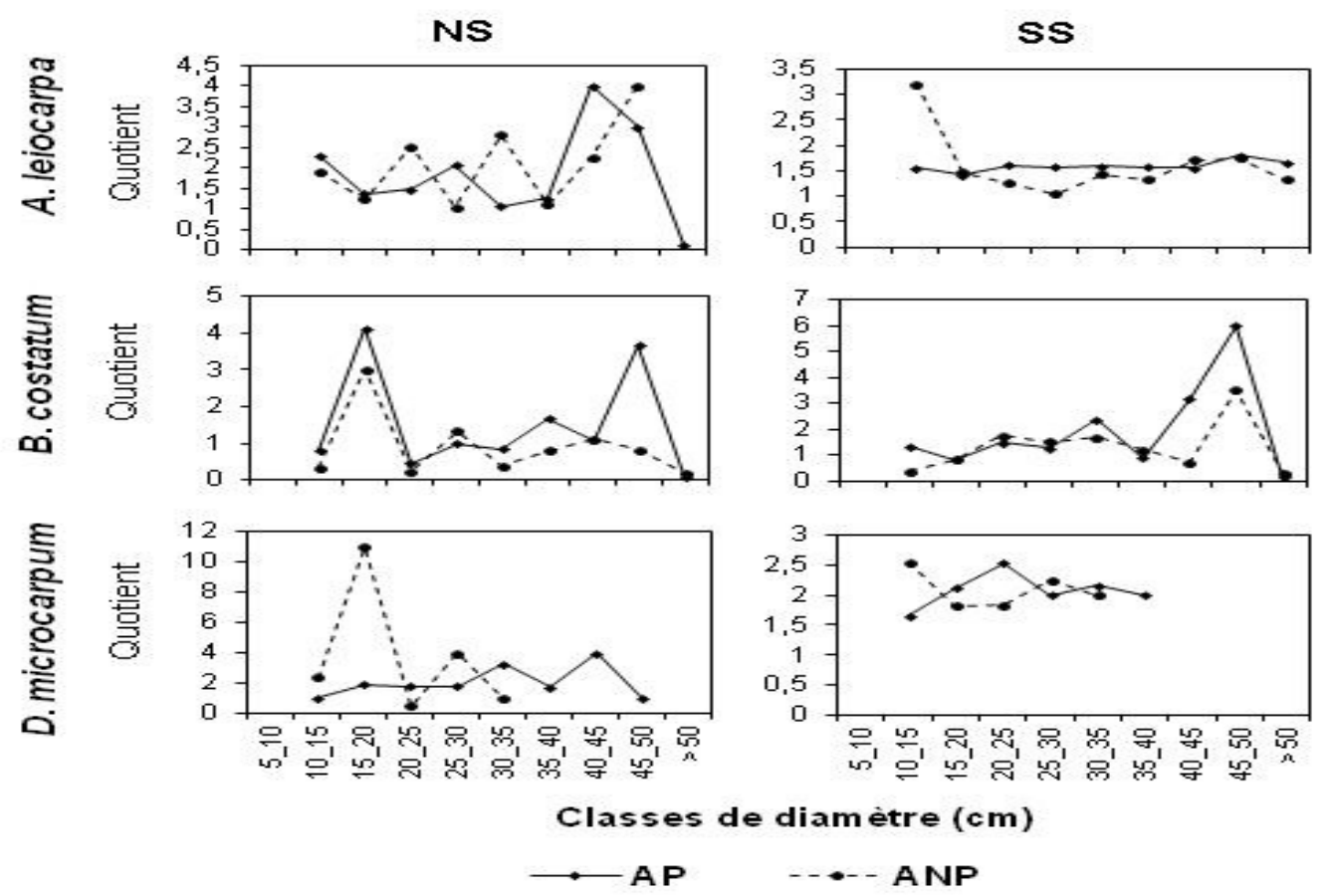

diam ètre $(\mathrm{cm})$

Figure 3 : Quotient entre les effectifs successifs des classes de diamètre de trois espèces (A. leiocarpa, B. costatum, et D. microcarpum) dans les aires protégées (AP) et dans les aires non protégées (ANP) du Nord-Soudanien (NS) et du Sud-Soudanien (SS). 
Tableau 4 : Comparaison des indicateurs de stabilité (pente et $\mathrm{r}^{2}$ ) des trois espèces sur chaque type d'utilisation des terres le long du gradient climatique.

\begin{tabular}{|c|c|c|c|c|c|c|c|c|c|}
\hline \multirow[b]{2}{*}{ Espèces } & \multirow[b]{2}{*}{ secteurs } & \multicolumn{4}{|c|}{ Aires protégées } & \multicolumn{4}{|c|}{ Aires non protégées } \\
\hline & & pentes & ET & $\mathbf{p}$ & $\mathbf{r}^{2}$ & pentes & ET & $\mathbf{p}$ & $\mathbf{r}^{2}$ \\
\hline \multirow[t]{2}{*}{ Anogeissus leiocarpa } & NS & $-1,24$ & 0,36 & \multirow{2}{*}{$\mathrm{p}>0,10$} & 0,67 & $-1,20$ & 0,46 & \multirow{2}{*}{$\mathrm{p}>0,10$} & 0,66 \\
\hline & SS & $-1,56$ & 0,42 & & 0,79 & $-1,08$ & 0,60 & & 0,65 \\
\hline \multirow[t]{2}{*}{ Bombax costatum } & NS & $-0,19$ & 0,52 & \multirow{2}{*}{$\mathrm{p}<0,10$} & 0,29 & 0,39 & 0,29 & \multirow{2}{*}{$\begin{array}{l}\mathrm{p}< \\
0,001\end{array}$} & 0,28 \\
\hline & SS & $-0,79$ & 0,51 & & 0,46 & $-0,23$ & 0,37 & & 0,23 \\
\hline \multirow[t]{2}{*}{ Detarium microcarpum } & NS & $-1,27$ & 0,51 & \multirow{2}{*}{$\mathrm{p}>0,10$} & 0,68 & $-1,08$ & 0,30 & \multirow{2}{*}{$\mathrm{p}>0,10$} & 0,73 \\
\hline & SS & $-1,64$ & 0,28 & & 0,80 & $-1,25$ & 0,29 & & 0,73 \\
\hline
\end{tabular}

ET $=$ Ecart type

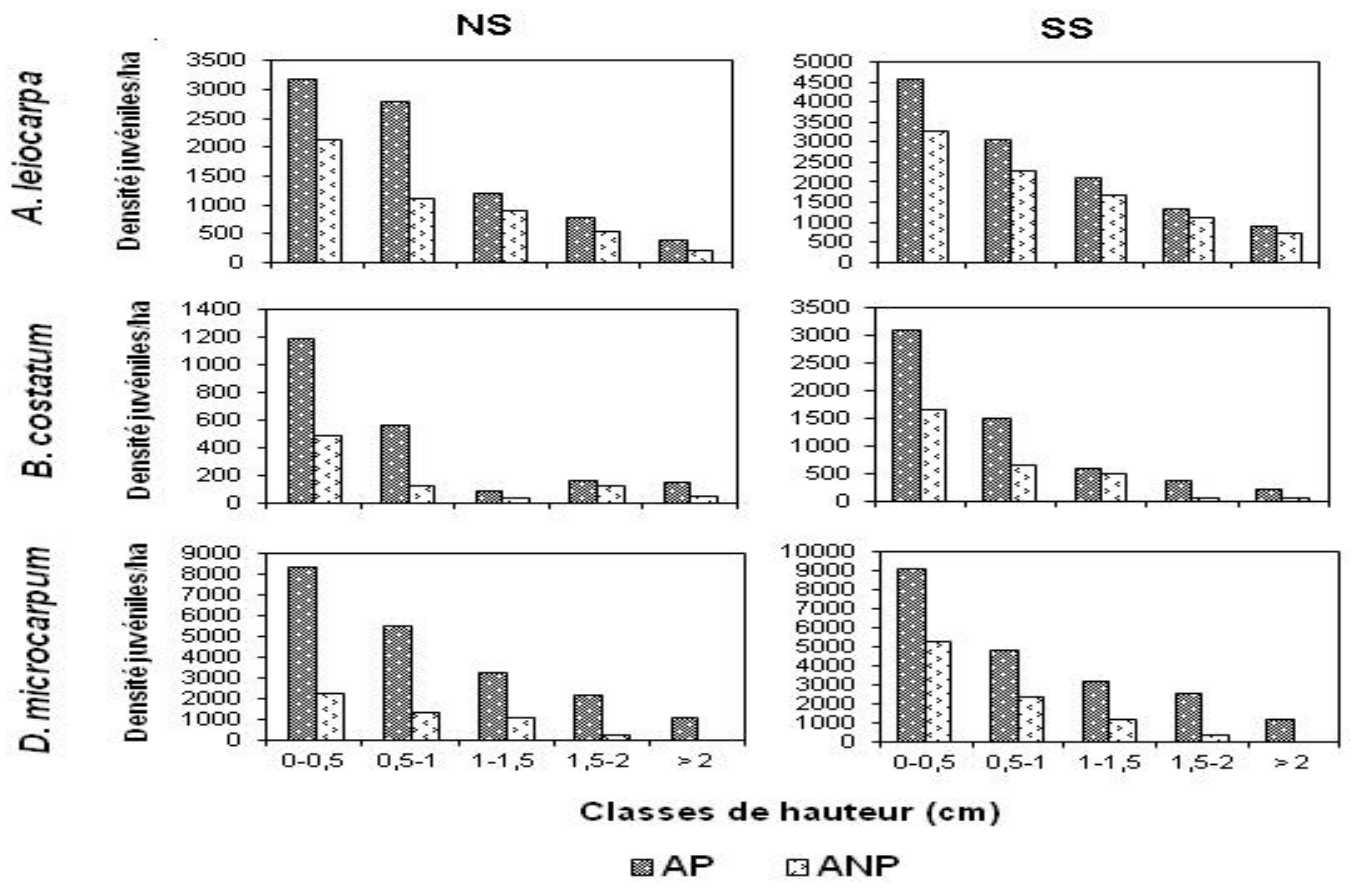

Figure 4 : Structures démographiques des populations juvéniles de trois espèces (A. leiocarpa, $B$. costatum et $D$. microcarpum) dans les aires protégées (AP) et dans les aires non protégées (ANP) du Nord-Soudanien (NS) et du Sud-Soudanien (SS). 
Tableau 5 : Résultats de l'analyse log-linéaire appliquée aux fréquences de classes de diamètre.

\begin{tabular}{|c|c|c|c|c|c|c|c|c|}
\hline \multirow{4}{*}{$\begin{array}{c}\text { Secteurs } \\
\text { phytogéogra } \\
\text { phiques }\end{array}$} & \multirow{4}{*}{ Espèces } & \multicolumn{7}{|c|}{ The CATMOD Procedure } \\
\hline & & \multicolumn{7}{|c|}{ Maximum Likelihood Analysis of Variance } \\
\hline & & \multicolumn{4}{|c|}{ Aires protégées } & \multicolumn{3}{|c|}{ Aires non protégées } \\
\hline & & Source & DF & Chi-Square & Pr $>$ ChiSq & DF & $\begin{array}{c}\text { Chi- } \\
\text { Square }\end{array}$ & $\begin{array}{c}\text { Pr }> \\
\text { ChiSq }\end{array}$ \\
\hline \multirow[t]{9}{*}{ Soudanien } & $\begin{array}{l}\text { Anogeissus } \\
\text { leiocarpa }\end{array}$ & class & 8 & 166,55 & $<0,0001$ & 6 & 71,68 & $<0,0001$ \\
\hline & & Distr & 1 & 0,07 & 0,7905 & 1 & 2,87 & 0,0840 \\
\hline & & class*Distr & 5 & 2,09 & $0,5539 *$ & 4 & 5,89 & $0,2355^{*}$ \\
\hline & $\begin{array}{l}\text { Bombax } \\
\text { costatum }\end{array}$ & class & 10 & 59,87 & $<0,0001$ & 8 & 23,97 & 0,0023 \\
\hline & & Distr & 1 & 0,14 & 0,7017 & 1 & 1,64 & 0,1867 \\
\hline & & class*Distr & 9 & 3,23 & $0,9282 *$ & 7 & 11,94 & $0,0837 *$ \\
\hline & $\begin{array}{l}\text { Detarium } \\
\text { microcarpum }\end{array}$ & class & 9 & 76,72 & $<0,0001$ & 9 & 9,51 & 0,3621 \\
\hline & & Distr & 1 & 5,73 & 0,0095 & 1 & 2,91 & 0,0894 \\
\hline & & class*Distr & 7 & 12,90 & $0,0843^{*}$ & 7 & 7,18 & $0,4131 *$ \\
\hline \multirow[t]{9}{*}{ Soudanien } & $\begin{array}{l}\text { Anogeissus } \\
\text { leiocarpa }\end{array}$ & class & 9 & 172,51 & $<0,0001$ & 9 & 106,14 & $<0,0001$ \\
\hline & & Distr & 1 & 1,45 & 0,2050 & 1 & 0,06 & 0,8463 \\
\hline & & class*Distr & 7 & 4,36 & $0,8012 *$ & 9 & 5,14 & $0,7761 *$ \\
\hline & $\begin{array}{l}\text { Bombax } \\
\text { costatum }\end{array}$ & class & 9 & 128,30 & $<0,0001$ & 9 & 104,31 & $<0,0001$ \\
\hline & & Distr & 1 & 0,91 & 0,3211 & 1 & 0,09 & 0,7971 \\
\hline & & class*Distr & 8 & 6,13 & $0,7326^{*}$ & 7 & 12,78 & $0,0856^{*}$ \\
\hline & $\begin{array}{l}\text { Detarium } \\
\text { microcarpum }\end{array}$ & class & 8 & 84,92 & $<0,0001$ & 9 & 51,72 & $<0,0001$ \\
\hline & & Distr & 1 & 0,00 & 0,9795 & 1 & 0,17 & 0,6761 \\
\hline & & class*Distr & 8 & 7,53 & $0,5708^{*}$ & 7 & 1,59 & $0,9824 *$ \\
\hline
\end{tabular}

*indique la valeur de l'interaction class*Distr qui montre que les structures observées s'ajustent à la distribution de Weibull 
Tableau 6 : Comparaison de la densité et des indicateurs de stabilité de la population juvénile des trois espèces entre les deux types d'utilisation des terres dans chaque secteur phytogéographique.

\begin{tabular}{|c|c|c|c|c|c|c|c|c|c|c|c|c|}
\hline Secteurs & Espèces & Statuts & $\begin{array}{l}\mathrm{Nb} \\
\text { relevé }\end{array}$ & $\begin{array}{l}\text { Densité } \\
\text { moyenne } \\
\text { (ind/ha) }\end{array}$ & ET & valeur $t$ & $\mathbf{p}$ & pentes & ET & $\mathbf{p}$ & $\mathrm{r}^{2}$ & PI \\
\hline \multirow[t]{7}{*}{ NS } & \multirow[t]{2}{*}{ A. leiocarpa } & $\mathrm{AP}$ & 11 & 12981,82 & 9612,26 & \multirow{2}{*}{2,58} & \multirow{2}{*}{$0,017 *$} & $-0,79$ & 0,24 & \multirow{2}{*}{$\mathrm{p}<0,10$} & 0,80 & 0 \\
\hline & & ANP & 11 & 4945,45 & 3723,53 & & & $-0,57$ & 0,15 & & 0,74 & 0 \\
\hline & \multirow[t]{2}{*}{ B. costatum } & $\mathrm{AP}$ & 20 & 2120 & 2164,20 & \multirow{2}{*}{2,30} & \multirow{2}{*}{$0,028^{*}$} & $-0,38$ & 0,53 & \multirow{3}{*}{$p>0,10$} & 0,55 & 2 \\
\hline & & ANP & 14 & 742,85 & 644,12 & & & $-0,30$ & 0,22 & & 0,62 & 6 \\
\hline & $D$. & & & & & & & & & & & \\
\hline & \multirow{2}{*}{ microcarpum } & $\mathrm{AP}$ & 14 & 20400 & 12168,05 & \multirow[t]{2}{*}{3,45} & \multirow[t]{2}{*}{$0,002 *$} & $-0,88$ & 0,20 & \multirow[t]{2}{*}{$p>0,10$} & 0,81 & 0 \\
\hline & & ANP & 8 & 5000 & 3806,76 & & & $-0,73$ & 0,23 & & 0,83 & 0 \\
\hline \multirow[t]{7}{*}{ SS } & \multirow[t]{2}{*}{ A. leiocarpa } & $\mathrm{AP}$ & 24 & 11983,33 & 9490,30 & \multirow{2}{*}{1,10} & \multirow{2}{*}{0,276} & $-0,57$ & 0,22 & \multirow{2}{*}{$p<0,10$} & 0,82 & 0 \\
\hline & & ANP & 19 & 9094,73 & 7092,83 & & & $-0,49$ & 0,11 & & 0,86 & 0 \\
\hline & \multirow[t]{2}{*}{ B. costatum } & $\mathrm{AP}$ & 16 & 5700 & 3411,35 & \multirow{2}{*}{1,84} & \multirow{2}{*}{0,078} & $-0,77$ & 0,28 & \multirow{2}{*}{$\mathrm{p}<0,10$} & 0,89 & 0 \\
\hline & & ANP & 8 & 2900 & 3714,06 & & & $-0,56$ & 0,37 & & 0,58 & 2 \\
\hline & \multirow{3}{*}{$\begin{array}{l}D . \\
\text { microcarpum }\end{array}$} & & & & & & & & & & & \\
\hline & & $\mathrm{AP}$ & 21 & 29857,14 & 18745,15 & \multirow[t]{2}{*}{2,20} & \multirow[t]{2}{*}{$0,034^{*}$} & $-0,84$ & 0,42 & \multirow[t]{2}{*}{$\mathrm{p}<0,05$} & 0,77 & 0 \\
\hline & & ANP & 14 & 9257,14 & 6942,14 & & & $-1,08$ & 0,29 & & 0,87 & 0 \\
\hline
\end{tabular}

Tableau 7 : Comparaison de la densité de la population juvénile des trois espèces sur chaque type d'utilisation de terres le long du gradient climatique.

\begin{tabular}{|c|c|c|c|c|c|c|c|c|c|}
\hline \multirow[b]{2}{*}{ Espèces } & \multirow[b]{2}{*}{ secteurs } & \multicolumn{4}{|c|}{ Aires protégées } & \multicolumn{4}{|c|}{ Aires non protégées } \\
\hline & & $\begin{array}{l}\text { Densité } \\
\text { moyenne } \\
\text { (ind/ha) }\end{array}$ & ET & valeur $\mathbf{t}$ & $\mathbf{p}$ & $\begin{array}{l}\text { Densité } \\
\text { moyenne } \\
\text { (ind/ha) }\end{array}$ & ET & valeur $\mathbf{t}$ & $\mathbf{p}$ \\
\hline \multirow[t]{2}{*}{ A. leiocarpa } & NS & 12981,82 & 9612,26 & \multirow{2}{*}{0,28} & \multirow{2}{*}{0,775} & 4945,45 & 3723,53 & \multirow{2}{*}{$-1,79$} & \multirow{2}{*}{0,083} \\
\hline & SS & 11983,33 & 9490,3 & & & 9094,74 & 7092,83 & & \\
\hline \multirow[t]{2}{*}{ B. costatum } & NS & 2120 & 2164,2 & \multirow{2}{*}{$-3,83$} & \multirow{2}{*}{$0,000^{*}$} & 742,85 & 644,16 & \multirow{2}{*}{$-2,15$} & \multirow{2}{*}{$0,043^{*}$} \\
\hline & SS & 5700 & 3411,35 & & & 2900 & 3714,06 & & \\
\hline \multirow[t]{2}{*}{ D. microcarpum } & NS & 20400 & 12168,05 & \multirow{2}{*}{$-0,08$} & \multirow{2}{*}{0,936} & 5000 & 3806,76 & \multirow{2}{*}{$-1,59$} & \multirow{2}{*}{0,127} \\
\hline & SS & 20857,14 & 18745,15 & & & 9257,14 & 6942,14 & & \\
\hline
\end{tabular}

\section{DISCUSSION}

Influence des types d'utilisation des terres et du climat sur l'état démographique des arbres

Cette étude permet de mettre en évidence l'impact de l'utilisation des terres et de la variation climatique sur les caractéristiques structurales des peuplements naturels des trois espèces. Les paramètres démographiques sont des indicateurs majeurs pour apprécier l'état et l'évolution qualitative et quantitative des peuplements forestiers (Oosterhoom and Kapelle, 2000 ; Kokou et al., 2005). Dans un même secteur climatique, la densité des peuplements est plus élevée dans les AP que les ANP; c'est le cas d'A. leiocarpa, de B. costatum et de D. microcarpum. Cette situation s'explique par le fait que dans les zones anthropisées, en plus de l'exploitation directe des espèces, les défriches 
agricoles contribuent à réduire considérablement l'étendu des peuplements de nombreuses espèces. L'impact négatif des différentes pressions anthropiques exercées sur les peuplements forestiers dans les régions soudano-sahéliennes de l'Afrique de l'Ouest a été largement documenté par de nombreux auteurs (Ouédraogo et al., 2006 ; Bouko et al., 2007 ; Diallo et al., 2011). Ces auteurs ont montré que les activités humaines telles que l'exploitation forestière, l'élevage, l'agriculture, l'expansion incontrôlée des habitations, les feux de brousse provoquent la destruction des écosystèmes forestiers et la disparition des espèces forestières. Ces pressions sont encore plus fortes sur certaines espèces à haute valeur socio-économique dont font partie A. leiocarpa, B. costatum et $D$. microcarpum (Lykke et al., 2004 ; Bognounou et al., 2009 ; Traoré et al., 2011).

L'effet de protection est plus perceptible sur l'état démographique de $D$. microcarpum dans le sud soudanien. Certes, toutes les aires protégées de la zone soudanienne ne sont pas entièrement à l'abri de l'action anthropique (Devineau, 2010), mais celles du sud soudanien semblent subir moins de pression d'exploitation compte tenu du milieu environnant qui offre des choix plus variés en termes de disponible ligneux à la faveur des conditions climatiques favorables. Dans le nord soudanien, la région de la boucle du Mouhoun abrite la plus importante proportion de population rurale du pays (INSD, 2008) et elle est aussi la plus intensément cultivée (Nikiéma et al., 2001). Il est reconnu que la pratique continue d'une agriculture extensive dans une région à forte densité de population contribue à détruire considérablement l'arbre et les formations forestières (Bouko et al., 2007 ; Nacoulma et al., 2011). Toutefois, les bonnes structures d'A. leiocarpa dans les zones anthropisées révèlent une capacité de résilience de l'espèce face aux pressions et cela est en accord avec les résultats obtenus dans la même zone soudanienne et à la même latitude, à l'Est du pays par Ouédraogo (2006) et Bognounou et al. (2009). La bonne adaptation de A. leiocarpa corrobore l'observation faite que l'espèce semble supporter la pression anthropique sous les conditions climatiques plus humides (Ouédraogo et al., 2006).

\section{Influence des types d'utilisation des terres et du climat sur la régénération naturelle}

La densité totale du peuplement juvénile de $B$. costatum dans les AP et dans les ANP montre une augmentation du NS au SS. Ce résultat est en accord avec ceux rapportés par Bognounou et al. (2010a) qui ont montré que la densité des ligneux augmente suivant le gradient du climat et se justifie par l'augmentation des précipitations. Par contre, A. leiocarpa et D. microcarpum ne montrent pas de différence significative au niveau de leur densité suivant le même gradient. Bien qu'une tendance à la hausse de densité soit observée au niveau de ces espèces, l'augmentation des précipitations du nord vers le sud soudanien ne semble pas influencer significativement l'abondance de leur peuplement juvénile. Cela traduit certainement le fait que ces deux espèces sont globalement bien adaptées au climat soudanien.

De nombreux facteurs peuvent réguler la régénération et le recrutement dans le peuplement adulte (Ryniker et al., 2006). Ces facteurs sont, entre autres, la faible production de semences la prédation des fruits, le pâturage, les feux de brousse, la luminosité sous le houppier et la variabilité du climat (Russell and Fowler, 2002). La mauvaise régénération naturelle des espèces est souvent évoquée comme une conséquence de la pénurie des semences (Weber et al., 2008; Bognounou et al., 2010a). Face aux contraintes environnementales, chaque espèce développe des stratégies pour assurer sa propre survie. En la matière, la morphologie fonctionnelle des plantules est un élément déterminant de l'adaptation aux conditions environnementales particulières (Bationo et al., 2010). C'est le cas de D. microcarpum qui a une germination cryptogée (Bationo et al., 2010) caractérisée par une soudure des cotylédons à la base et l'enfouissement du collet dans le sol (Bationo et al., 2001a), ce qui permet aux bougeons cotylédonaires situés dans la zone du collet d'être à l'abri des intempéries (Bationo et al., 2001b). Le pivot racinaire de D. microcarpum a une croissance rapide, ce qui facilite son 
enracinement dans le sol (Bationo et al., 2010). Ces fonctionnements écophysiologiques pourraient expliquer en grande partie l'adaptation des plantules cryptogées de cette espèce au régime des feux tardifs dans les savanes ouvertes et au pâturage. La spécialisation du système racinaire en racine tubérisée permet également à cette espèce de stocker des réserves d'eau et d'autres substances nutritives et de survivre à la destruction des parties aériennes dues au traumatisme divers sous forme de plantules géophytes (Bationo et al., 2010). De même, les plantules B. costatum sont capables de développer des racines tubérisées qui permettent de stocker de l'eau et des nutriments pour passer la mauvaise saison (Ouédraogo and Thiombiano, 2012).

La récolte des fleurs se fait parfois souvent par écimage presque complet du houppier (Belem et al., 2007), réduisant ainsi considérablement le potentiel semencier des arbres chez B. costatum (Belem et al., 2008). En zones anthropisées, les peuplements de $B$. costatum présentent de mauvaises structures caractérisées par une réjuvénilisation nulle ou déficitaire (Belem et al., 2008 ; Ouédraogo and Thiombiano, 2012). La reproduction par voie sexuée est quasi absente et c'est le drageonnage qui est le principal mécanisme de régénération chez l'espèce (Ouédraogo et al., 2006). Ce mécanisme de régénération serait donc à l'origine de la relative bonne régénération de l'espèce, beaucoup plus perceptible dans le sud soudanien.

Quand à A. leiocarpa, sa régénération naturelle est principalement assurée par la reproduction asexuée dont les rejets de collet et les rejets de souche sont les principaux mécanismes (Bognounou et al., 2010a). La dominance du recrutement végétatif de cette espèce reflète plutôt une dynamique de survie ou de surpassement de ses capacités de reproduction sexuée (Ouédraogo, 2006), car le taux de germination de ses graines est très faible (Thiombiano et al., 2003 ; Dayamba et al., 2008 ; Bognounou et al., 2010b), à cause d'une grande proportion de fruits infertiles (Thiombiano, 2005).

\section{Conclusion}

Les résultats de la présente étude montrent que les types d'utilisation des terres et la variabilité climatique entre les secteurs phytogéographiques nord et sud soudanien ont une influence sur les caractéristiques démographiques des trois espèces. Toutefois, la sensibilité aux facteurs climatiques et anthropiques est variable suivant les espèces. L'influence du climat sur les caractéristiques démographiques des trois espèces dans les AP est seulement perceptible sur $B$. costatum mais elle ne l'est pas sur ces mêmes espèces dans les ANP, révélant ainsi une forte pression anthropique sur les zones ouvertes. Les caractéristiques démographiques et dendrométriques varient suivant les types d'utilisation des terres, ce qui tend à confirmer une partie de notre hypothèse à savoir que les types d'utilisation des terres influencent significativement la structure des trois peuplements. L'évaluation de l'avenir des populations des trois espèces à travers l'étude de la régénération montre de bonnes dispositions démographiques pour A. leiocarpa et $D$. microcarpum sur les types d'utilisation des terres, dues à leurs stratégies d'adaptation fonctionnelles aux conditions environnementales de plus en plus difficiles.

La conservation et la gestion durable de ces trois espèces vulnérables exigent le renforcement des mesures de protection des aires protégées et une assistance de la régénération qui consisterait au moins à protéger les jeunes plants durant les premières années de leur croissance. Cela pourrait être envisagé par la réalisation des pares-feux et le respect strict des périodes d'utilisation des feux d'aménagement (précoces) pour réduire leur impact destructif sur la régénération.

\section{CONFLIT D'INTERETS}

Les auteurs de ce manuscrit déclarent qu'il n'y a aucun conflit d'intérêt entre eux.

\section{CONTIBUTIONS DES AUTEURS}

Dans le cadre de la réalisation de cette étude, LT a élaboré le protocole de recherche sous la supervision de AT, collecté et traité les données et aussi rédigé le manuscrit. OS et SS 
ont participé à la rédaction du manuscrit. $\mathrm{AO}$ et AT ont participé à la relecture du document. Tous les auteurs ont approuvé la version finale du manuscrit.

\section{REMERCIEMENTS}

Les auteurs remercient le ministère fédéral allemand de l'Éducation et de la Recherche (BMBF) qui a financé cette étude à travers le Programme BIOTA West. Ils remercient également Monsieur Abel Kadéba pour la réalisation de la carte de la zone d'étude et tous les pisteurs commis à la tâche de guide de terrain.

\section{REFREENCES}

Arbonnier M. 2002. Arbres, Arbustes et Lianes des Zones Sèches d'Afrique de l'Ouest. Editions CIRAD-MNHN-2ème édition : Paris ; 573.

Aké Assi L. 2001. Flore de Côte d'Ivoire: Catalogue Systématique, Biogéographie et Ecologie I. Conservatoire et Jardin Botaniques de Genève: Genève, Boissiera 57 ; 396.

Ariori SL, Ozer P. 2005. Évolution des ressources forestières en Afrique de l'Ouest soudano-sahélienne au cours des 50 dernières années. International Journal of Tropical Geology, Geography and Ecology, 29: 61-68. DOI: http://hdl.handle.net/2268/15851

Baker PJ, Bunyavejchewin S, Oliver CD, Ashton PS. 2005. Disturbance history and historical stand dynamics of a seasonal tropical forest in western Thailand, Ecological Monographs, 75(3): 317-343. DOI: 10.1890/04-0488

Bationo BA, Ouédraogo SJ, Guinko S. 2001a. Stratégies de régénération naturelle de Detarium microcarpum Guill. et Perr. dans la forêt classée de Nazinon (Burkina Faso), Fruits, 56: 271-285. DOI: 10.1051/fruits:2001129

Bationo BA, Ouédraogo SJ, Alexandre DY, GuinkoS. 2001b. Statut hydrique de quatre espèces ligneuses soudaniennes dans la forêt de Nazinon, Burkina Faso, Sécheresse, $\quad \mathbf{1 2}(2)$ : $\quad 87-94$. http://horizon.documentation.ird.fr/exl- doc/pleins_textes/pleins_textes_7/b_fdi_ 59-60/010025746.pdf

Bationo BA, Somé NA, Ouédraogo SJ, Kalinganire A. 2010. Croissance comparée des plantules de cinq espèces ligneuses soudaniennes élévées en rhizotron. Sécheresse, 21(3): 196-202. DOI : $10.1684 / \mathrm{sec} .2010 .0255$

Bastide B, Ouédraogo SJ. 2008. Rejets de Detarium microcarpum et feux précoces. Bois et Forêts des Tropiques, 296(2) : 2737. DOI: https://doi.org/10.19182/bft2008.296.a20 381

Belem B, Nacoulma BMI, Gbangou R, Kambou S, Hansen HH, Gausset Q, Lund S, Raebild A, Lompo D, Ouédraogo M, Theilade I, Boussim JI. 2007. Use of None Wood Forest Products by local people bordering the "Parc National Kaboré Tambi", Burkina Faso. The Journal Transdisciplinary Environmental Studies, 1: 1-21.

Belem B, Boussim JI, Bellefontaine R, Guinko S. 2008. Stimulation du drageonnage de Bombax costatum par blessure des racines au Burkina Faso. Bois et Forêts des Tropiques, 295: 71-79. http://publications.cirad.fr/une_notice.ph $\mathrm{p} ? \mathrm{dk}=542832$

Belemsobgo U, Kafando P, Adouabou BA, Nana S, Coulibaly S, Gnoumou A, Konrad T. 2010. Le réseau d'Aires Protégées. In Atlas de la Biodiversité de l'Afrique de l'Ouest, Tome II : Burkina Faso. Ouagadougou \& Frankfurt/Main ; 354-363.

Bénoit E. 2008. Les changements climatiques : vulnérabilité, impacts et adaptation dans le monde de la médecine traditionnelle au Burkina Faso. VertigO, 1 : 1-12. DOI: https://doi.org/10.4000/vertigo.1467

Bognounou F. 2009. Restauration écologique et gradient latitudinal: utilisation, diversité et régénération de cinq espèces de Combretaceae au Burkina Faso, Thèse de Doctorat unique, Université de Ouagadougou, p. 179.

Bognounou F, Thiombiano A, Savadogo P, Boussim JI, Odén PC, Guinko S. 2009. Woody vegetation structure and 
composition at four sites along latitudinal gradient in Western Burkina Faso, Bois et Forêts des Tropiques, 300: 29-44. DOI: 10.19182/bft2009.300.a20412

Bognounou F, Tigabu M, Savadogo P, Thiombiano A, Boussim JI, Oden PC, Guinko S. 2010a. Regeneration of five Combretaceae species along a latitudinal gradient in Sahelo-Sudanian zone of Burkina Faso. Annals of Forest Science, 67. DOI: $10.1051 /$ forest/2009119

Bognounou F, Thiombiano A, Odén PC, Guinko S. 2010b. Seed provenance and latitudinal gradient effects on seed germination capacity and seedling establishment of five indigenous species in Burkina Faso. Tropical Ecology, 51(2): 207-220.

https://www.cabdirect.org/cabdirect/abst ract/20103143284

Bonou W, Glele Kakaï R, Assogbadjo AE, Fonton HN, Sinsin B. 2009. Characterisation of Afzelia africana Sm. Habitat in the Lama forest reserve of Benin. Forest Ecology and Management, 258: $\quad$ 1084-1092. DOI: https://doi.org/10.1016/j.foreco.2009.05. 032

Botha J, Witkowski ETF, Shackleton CM. 2004. Harvesting impacts on commonly used medicinal tree species (Catha edulis and Rapanea melanophloeos) under different land management regimes in the Mpumalanga Lowveld, South Africa, Koedoe, 47: 1-18. DOI: https://doi.org/10.4102/koedoe.v47i2.77

Bouko BS, Sinsin B, Soule BG. 2007. Effets de la dynamique d'occupation du sol sur la structure et la diversité floristique des forêts claires et savanes au Bénin, Tropicultura, 4 : 221-227.

Bunasols. 2002a. Etude morphopédologique des provinces $d u$ Yatenga, du Loroum et $d u$ Zondoma. Echelle : 1/100 000. Ouagadougou p. 122.

Bunasols. 2002b. Etude morphopédologique des provinces du Sourou et du Nayala. Echelle : 1/100 000, p.123.

Bunasols. 2002c. Etude morphopédologique de la province du Soum. Echelle : 1/100 000, $125 \mathrm{p}$.
Bunasols. 2006. Etude morphopédologique des provinces du Mouhoun et des Ballés. Echelle : 1/100 000. Ouagadougou, p. 135.

Caswell H. 2001. Matrix Population Models: Construction Analysis and Interpretation 2nd ed. Sinauer Associates, Sunderland, Massachusetts.

Chappell A, Agnew CT. 2004, Modelling climate change in West African Sahel rainfall (1931-1990) as an artifact of changing station locations. International Journal of Climatology, 24: 547-554. DOI: https://doi.org/10.1002/joc.1021

Condit R, Sukumar R, Hubbell SP, Foster RB. 1998. Predicting population trends from size distribution: a direct test in a tropical tree community. American Naturalist, 152(4): 495-509. DOI: 10.1086/286186.

Dai A, Lamb PJ, Trenberth KE, Hulme M, Jones PD, Xie P. 2004. The Recent Sahel drought is real. International Journal of Climatology, 24: 1323-1331. DOI: 10.1002/joc. 1083

Dayamba SD, Tigabu M, Sawadogo L, Oden PC. 2008. Seed germination of herbaceous and woody species of the Sudanian savanna-woodland in response to heat shock and smoke. Forest Ecology and Management, 256 : 462-470. DOI: 10.1016/j.foreco.2008.04.051

Diallo H, Bamba I, Sadaiou Y, Barima S, Visser M, Ballo A, Mama A, Vranken I, Maiga M, Bogaert J. 2011. Effets combinés du climat et des pressions anthropiques sur la dynamique évolutive de la végétation d'une zone protégée du Mali (Réserve de Fina, Boucle du Baoulé). Sécheresse, 2 : 97-107. DOI : 10.1684/sec.2011.0306

Devineau JL. 2010. To what extent does landuse affect relationships between the distribution of woody species and climatic change? A case study along an aridity gradient in western Burkina Faso, Plant Ecology, 212: 959-973. DOI: 10.1007/s11258-010-9877-8

Driessen P, Deckers J, Spaargaren O. 2001. Lecture notes on the major soils of the world. Rome: Food and Agriculture 
Organization of the United Nations, p.334.

Everard DA, Midgley JJ, Van Wyk GF. 1995. Dynamics of some forests in KwaZuluNatal, South Africa, based on ordinations and size class distribution, South African Journal of Botany, 61: 283-292. DOI: https://doi.org/10.1016/S02546299(15)30548-2

Folelack DP, Abou S. 2009. Commercialisation du bois de chauffe en zone sahélienne du Cameroun. Sécheresse, 3 : 312-318.

Fontès J, Guinko S. 1995. Carte de la végétation et de l'occupation du sol (Burkina Faso). Notice explicative. Laboratoire d'Écologie Terrestre, Institut de la Carte Internationale de la Végétation. CNRS, Université de Toulouse 3 (France). Institut du Développement Rural, Faculté des Sciences et Techniques, Université de Ouagadougou, Burkina Faso. Ministère de la Coopération française, Projet Campus

Ganaba S, Ouadba JM, Bognounou O. 2004. Plantes de construction d'habitations en région Sahélienne. Bois et Forêts des Tropiques, 4 : 11-17.

Gaoue OG, Ticktin T. 2008. Impacts of bark and foliage harvest on Khaya senegalensis (Meliaceae) reproductive performance in Benin. Journal of Applied Ecology, 45: 34-40. DOI: 10.1111/j.1365-2664.2007. 01381.x

INSD (Institut National de la Statistique et de la Démographie). 2008. Recensement Général de la Population et de l'Habitation de 2006, Résultats définitifs, Burkina Faso, Ouagadougou.

Johnson NL, Kotz S. 1970. Distributions in Statistics: Continuous Univariate Distributions. John Wiley and Sons: New York, USA.

Kagoné H. 2001. Profile Fourrager, Burkina Faso. Collection Grassland and Pasture Crops, FAO, p. 27.

Kokou K, Adjossou K, Hamberger K. 2005. Les forêts sacrées de l'aire Ouatchi au Sud-Est du Togo et les contraintes actuelles des modes de gestion locales des ressources forestières. $\operatorname{Vertig} O$, 3: 1-10. DOI:

https://doi.org/10.4000/vertigo.2456

Lorimer CG, Krug AG. 1983. Diameter distribution in even-aged stands of shadetolerant and midtolerant tree species, American Midland Naturalist, 109: 331345.

DOI: https://doi.org/10.2307/2425414

Lykke AM. 1998. Assessment of species composition change in savanna vegetation by means of woody plants' size class distribution and local information. Biodiversity and Conservation, 7: 1261-1275. DOI: https://doi.org/10.1023/A:100887781928 6

Lykke AM, Kristensen MK, Ganaba S. 2004. Valuation of local use and dynamics of 56 woody species in the Sahel. Biodiversity and Conservation, 13: 1961-1990. DOI: https://doi.org/10.1023/B:BIOC.0000035 876.39587.1a

Mertz O, Lykke AM, Reenberg A. 2001. Importance and seasonality of vegetable consumption and marketing in Burkina Faso. Economic Botany, 55 : 276-289. DOI: https://doi.org/10.1007/BF02864565.

Mwavu EN, Witkowski ETF. 2009. Population structure and regeneration of multiple-use tree species in a semi-deciduous African tropical rainforest: Implication for primate conservation. Forest and Ecology Management, 258: 840-849. DOI: 10.1016/j.foreco.2009.03.019

Nacoulma BMI, Schumann K, Traoré S, Bernhardt-Römermann $\mathrm{M}$, Hahn $\mathrm{K}$, Wittig R, Thiombiano A. 2011. Impacts of land-use on West African savanna vegetation: a comparison between protected and communal area in Burkina Faso. Biodiversity and Conservation, DOI: 10.1007/s10531-011-0114-0.

Nicholson SE. 2005. On the question of the "recovery" of the rains in the West African Sahel. Journal of Arid Environments, 63: 615-641. DOI: 10.1016/j.jaridenv.2005.03.004

Nikiéma A, Ouédraogo SJ, Boussim JI. 2001. Situation des ressources génétiques 
forestières du Burkina Faso, Atelier sousrégional FAO/IPGRI/ICRAF sur la conservation, la gestion, l'utilisation durable et la mise en valeur des ressources génétiques forestières de la zone sahélienne (Ouagadougou, 22-24 sept. 1998), Note thématique sur les ressources génétiques forestières, Document FGR/22F, Département des forêts, FAO, Rome, Italie.

Obiri J, Lawes M, Mukolwe M. 2002. The dynamics and sustainable use of highvalue tree species of the coastal Pondoland forest of the Eastern Cape Province South Africa. Forest Ecology and Management, 166: 131-148. DOI: 10.1016/S0378-1127(01)00665-X

Oosterhoorn M, Kapelle M. 2000. Végétation structure and composition along an interior-edge-exterior gradient in a Costa Rican montane cloud forest. Forest Ecology and Management, 3: 291-307. DOI: 10.1016/S0378-1127(99)00101-2

Ouédraogo A. 2006. Diversité et dynamique de la végétation ligneuse de la partie orientale du Burkina Faso, Thèse de Doctorat unique, Université de Ouagadougou, p.196.

Ouédraogo A, Thiombiabo A, Hahn-Hadjali K, Guinko S. 2006. Diagnostic de l'état de dégradation des peuplements de quatre espèces ligneuses en zone soudanienne du Burkina Faso. Sécheresse, 4 : 485-491.

Ouédraogo A, Thiombiano A. 2012. Regeneration pattern of four threatened tree species in Sudanian savannas of Burkina Faso. Agroforestry Systems, DOI 10.1007/s10457-012-9505-9

Ouédraogo I, Nacoulman BMI, Ouédraogo O, Hahn K, Thiombiano A. 2014.

Productivité et valeur économique des calices de Bombax costatum Pellegr. \& Vuillet en zone soudanienne du Burkina Faso. Bois et Forêts des Tropiques, 319 (1) : 31-41.

Ouôba P, Lykke AM, Boussim JI, Guinko S. 2006. La flore médicinale de la Forêt Classée de Niangoloko (Burkina Faso). Etude sur la Flore et la Végétation du Burkina Faso et des Pays Avoisinants, 10 : 5-16.
Ozer P, Hountondji YC, Niang AJ, Karimoune S, Laminou Manzo O, Salmon M. 2010. Désertification au Sahel: Historique et perspectives. BSGLg, 54: 69-84. http://hdl.handle.net/2268/10347

Rondeux J. 1999. La Mesure des Peuplements Forestiers (2 édn). Presses Agronomiques de Gembloux : Gembloux ; 544.

Russell FL, Fowler NL. 2002. Failure of adult recruitment in Quercus buckleyi populations on the eastern Edwards Plateau, Texas. American Midland Naturalist, $\quad$ 148: 201-217. https://www.jstor.org/stable/3083125

Ryniker KA, Bush JK, Van Auken OW. 2006. Structure of Quercus gambelii communities in the Lincoln National forest, New Mexico, USA. Forest Ecology and Management, 233: 69-77. DOI: $10.1016 /$ j.foreco.2006.06.008

Sambaré O, Bognounou F, Wittig R, Thiombiano A. 2011. Woody species composition, diversity and structure of riparian forests of four watercourses types in Burkina Faso, Journal of Forestry Research, 22: 145-158. https://doi.org/10.1007/s11676-0110143-2

Sawadogo H, Zombré PN, Bock L, Lacroix D. 2008. Évolution de l'occupation du sol de Ziga dans le Yatenga (Burkina Faso) à partir de photos aériennes. Revue de Télédétection, $\quad \mathbf{8}: \quad$ 59-73. https://halshs.archivesouvertes.fr/halshs-00386400

Shackleton CM. 1993. Demography and dynamics of the dominant woody species in a communal and protected area of the eastern Transvaal Lowveld. South African Journal of Botany, 59: 569-574. DOI: https://doi.org/10.1016/S02546299(16)30672-X

Sop TK, Oldeland J, Schmiedel U, Ouédraogo I, Thiombiano A. 2010. Population structure of three woody species in four ethnic domains of the sub-sahel of Burkina Faso. Land Degradation and Development, DOI: 10.1002/ldr.1026

Sop TK, Oldeland J, Bognounou F, Schmiedel U, Thiombiano A. 2012. Ethnobotanical 
knowledge and valuation of woody plants species: a comparative analysis of three ethnic groups from the sub-Sahel of Burkina Faso. Environment, Development and Sustainability, 14 : 627-649. DOI : 10.1007/s10668-0129345-9.

Tabuti JRS. 2007. The uses, local perceptions and ecological status of 16 woody species of Gadumire Sub-county, Uganda. Biodiversity and Conservation, 16: 19011915. DOI: 10.1007/s10531-006-9097-7

Thiombiano A, Wittig R, Guinko S. 2003. Conditions de la multiplication sexuée chez les Combretaceae du Burkina Faso. Revue d'Ecologie-La Terre Et La Vie, 58 : 361-379. http://hdl.handle.net/2042/55562

Thiombiano A. 2005. Les Combretaceae du Burkina Faso: taxonomie, écologie, dynamique et régénération des espèces, Thèse de Doctorat d'Etat, Université de Ouagadougou, p. 290.

Thiombiano A, Schmidt M, Kreft H, Guinko S. 2006. Influence du gradient climatique sur la distribution des espèces de Combretaceae au Burkina Faso (Afrique de l'Ouest), Candollea, 61: 189-213. http://www.ville-

ge.ch/cjb/publications/cando611/candoll ea61-1_189-213

Tiétiambou FRS, Lykke AM, Korbéogo G, Thiombiano A, Ouédraogo A. 2016. Perceptions et savoirs locaux sur les espèces oléagineuses locales dans le Kénédougou, Burkina Faso, Bois et Forêts des Tropiques, 327(1) : 39-50.

Traoré L, Ouédraogo I, Ouédraogo A, Thiombiano A. 2011. Perceptions, usages et vulnérabilité des ressources végétales ligneuses dans le Sud-Ouest du Burkina Faso. International Journal of Biological and Chemical Sciences, 5(1): 258-278. DOI: 10.4314/ijbcs.v5i1.68103 .

Traoré L, Sop TK, Dayamba SD, Traoré S, Hahn K, Thiombiano A. 2013. Do protected areas really work to conserve ? A case study of three vulnerable woody species in the Sudanian zone of Burkina Faso. Environment Development and Sustainability, 15(3): 663-686. DOI: 10.1007/s10668-012-9399-8

Venter SM, Witkowski ETF. 2010. Baobab (Adansonia digitata L.) density, sizeclass distribution and population trends between four land-use types in northern venda, South Africa. Forest Ecology and Management, 259: 294-300. DOI: 10.1016/j.foreco.2009.10.016

Weber JC, Larwanou M, Abasse TA, Kalinganire A. 2008. Growth and survival of Prosopis africana provenances tested in Niger and related to rainfall gradients in the West African Sahel. Forest Ecology and Management, 256: $585-592 . \quad$ DOI: 10.1016/j.foreco.2008.05.004

Wiegand K, Ward D, Thulke H, Jeltsch F. 2000. From snapshot information to longterm population dynamics of Acacias by a simulation model. Plant Ecology, 150: 97-114. DOI: 10.1023/A:1026574303048

Zarnock SJ, Dell TR. 1985. An evaluation of percentile and maximum likelihood estimators of Weibull parameters, Forest Sciences, 31: 260-268. 